\title{
Smooth Tests of Copula Specifications
}

\author{
Juan Lin* Ximing $\mathrm{Wu}^{\dagger}$
}

December 24, 2012

\begin{abstract}
We present a family of smooth tests for the goodness of fit of semiparametric multivariate copula models. The proposed tests are distribution free and can be easily implemented. They are diagnostic and constructive in the sense that when a null distribution is rejected, the test provides useful pointers to alternative copula distributions. We then propose a method of copula density construction, which can be viewed as a multivariate extension of Efron and Tibshirani (1996). We further generalize our methods to dynamic copula models of Chen and Fan (2006). We report extensive Monte Carlo simulations and three empirical examples to illustrate the effectiveness and usefulness of our method.
\end{abstract}

Keywords: copula specification; smooth test; empirical copula distributions

\section{Introduction}

The method of copula has been extensively used in risk management, finance and insurance due to its flexibility in separately modeling the marginal distributions of individual series and their dependence structure, cf. Cherubini et al. (2004, 2011), McNeil et al. (2005), Patton (2012) and references therein. Commonly used copulas are often parameterized by one or two parameters. The Gaussian copula is most popular for it is easy to compute, simulate and can be easily extended to arbitrary dimensions. The Gaussian copula is also easy to calibrate since it is uniquely defined by the correlation matrix of marginal distributions and therefore only requires calculating pairwise correlations. However, its simplicity and ease of use come with a price. The Gaussian copula has several drawbacks. For instance, it does not allow tail dependence and therefore cannot capture interdependence among extreme events. In addition, the Gaussian copula is radially symmetric and thus does not allow asymmetric dependence among variables.

It is widely appreciated that specification testings of parametric copula model are of great importance since different parametric copulas lead to multivariate models that may have very

${ }^{*}$ Risk Management Institute, National University of Singapore. email: rmilj@nus.edu.sg

${ }^{\dagger}$ Department of Agricultural Economics, Texas A\&M University. email: xwu@tamu.edu 
different dependence properties. A number of existing papers have attempted to address this issue, including among others, Chen et al. (2004), Chen and Fan (2005, 2006), Fermanian (2005), Chen (2007), Li and Peng (2009), Prokhorov and Schmidt (2009), Chen et al. (2010), and Manner and Reznikova (2012). For general overviews of copula specification tests, see Genest et al. (2009), Berg (2009), and Fermanian (2012). The majority of tests reviewed in these survey articles are omnibus or 'blanket' tests which possess powers against all alternatives.

Although omnibus tests are known to be consistent, they often only possess satisfactory power against certain deviations from the null hypothesis and lack power in specific directions (Janssen, 2000). Moverover, they might not have good finite sample power properties (Bera et al., 2012). Recently, Chen (2007) proposes moment based copula tests for multivariate dynamic models whose marginals and copulas are both parametrically specified. Chen (2007) indicates that "although the moment-based tests have better size performance, they require correctly specified standardized error distributions." In practice, it is rare that the true marginal distributions are known. Consequently, tests based on parametrically estimated marginal distributions may have size distortions. It is conceivable that moment-based tests constructed upon empirical CDF's should combine the strength of moment-based tests and the robustness of empirical CDF's to offer a viable alternative. In this paper, we aim to fill this gap in the literature by proposing a family of moment-based copula tests that use empirical CDF's of the marginal distributions. Our tests are distribution free and can be easily calculated. They can be viewed as a generalization of the smooth test of Neyman (1937). Unlike omnibus tests, moment-based tests face the nontrivial task of moment selection. We base our tests on a series of orthogonal basis functions such that the selection of moments can be proceeded hierarchically. At the same time, the design of tests is flexible enough such that they can be tailored to focus on certain types of hypotheses, such as symmetry, tail dependence and so on. Results from Monte Carlo simulations demonstrate good finite sample size and power performance of our tests.

The proposed tests have a particularly appealing feature. When a null hypothesis is rejected under a selected set of moment conditions, we can subsequently construct alternative copula functions by augmenting the null distribution with these extra moment conditions in the spirit of Efron and Tibshirani (1996). Therefore, the tests are not only diagnostic but also conductive to improved copula specifications. Our numerical examples illustrate the usefulness of this approach: the augmented copula functions are shown to provide more satisfactory fitting to the dependence structure among variables of interest.

Copula methods have found most applications in financial economics, wherein the data often exhibit inter temporal dependence such as series correlation or clusters of extreme values. To accommodate time series data, we further extend our methods to the semiparametric copula-based multivariate dynamic (SCOMDY) models as in Chen and Fan (2006). A key finding of Chen and Fan (2006) is that the copula parameters are asymptotically invariant to the estimation of dynamic parameters when the marginal distributions are estimated by the empirical CDF's. This 
remarkable asymptotic invariance is confirmed in our simulations. We show that our tests extend to the innovations of SOCMDY models directly and possess the same good finite sample performance as in the iid case.

The remainder of this paper is organized as follows. In sector 2, we first briefly review Neyman's smooth test of distributions and then present copula smooth tests under simple hypotheses. In Section 3, we present general copula smooth tests with nonparametric marginal distributions and estimated copula parameters. In section 4, we extend the tests to copula-based multivariate dynamic models. Finite sample performance of the proposed tests are investigated in section 5 through Monte Carlo simulations. Section 6 provides three empirical applications and the last section briefly concludes. All proofs are relegated to the appendices.

Throughout the paper, we use upper case letters to denote cumulative distribution functions and corresponding lower case letters to denote the density functions. We use subscript $t$ to index observations and subscript $j$ to denote the coordinate of multivariate random vectors. For simplicity, we focus on bivariate copulas in this study. Extensions to higher dimensional cases are straightforward.

\section{Copula Tests under Simple Hypotheses}

In this section, we present smooth tests of copula specifications under the ideal situation of known marginal distributions and known copula distributions. Since our tests are motivated by Neyman's smooth test of distributions, we first provide a brief introduction of Neyman's test, followed by our smooth tests of copula distributions under simple hypotheses.

\subsection{Neyman's Smooth Test}

Let $Y_{1}, \ldots, Y_{n}$ be an iid random sample from an unknown distribution. The hypothesis of interest is to test whether the sample is generated from a specific distribution $F_{y}$. Neyman's test of distribution is based on the fact that under the null $F_{y}\left(Y_{1}\right), \ldots, F_{y}\left(Y_{n}\right)$ converge in distribution to the standard uniform distribution as $n \rightarrow \infty$. Therefore, the test on a generic distribution $F_{y}$ is transformed to a test on uniformity. Neyman (1937) motivates his smooth test with following smooth alternative to the uniform density

$$
f_{K}(v)=\exp \left(\sum_{k=1}^{K} \lambda_{k} \psi_{k}(v)-\lambda_{0}\right), v \in[0,1],
$$

where $\lambda_{0}=\log \int \exp \left(\sum_{k=1}^{K} \lambda_{k} \psi_{k}(v)\right) d v$ ensures that $f$ integrates to unity, and $\psi_{k}$ 's are normalized Legendre polynomials on $[0,1]$ given by

$$
\psi_{k}(v)=\frac{(k !)^{-1}}{\sqrt{2 k+1}} \frac{d^{k}}{d v^{k}}\left\{\left(v^{2}-v\right)^{k}\right\}
$$


which are orthonormal with respect to the standard uniform distribution.

Under the null, $\lambda \equiv\left(\lambda_{1}, \ldots, \lambda_{K}\right)^{T}=0$ and the test of uniformity is equivalent to a test on $\lambda=0$. One can construct a likelihood ratio test on this hypothesis. Alternatively one can use a score test. Although asymptotically equivalent to the likelihood ratio test, the score test is locally optimal and particularly appealing since it does not require the estimation of $\lambda$. Let $\psi=\left(\psi_{1}, \ldots, \psi_{K}\right)^{T}$ and

$$
\hat{\psi}=\frac{1}{n} \sum_{t=1}^{n} \psi\left(F_{y}\left(Y_{t}\right)\right) .
$$

Neyman's smooth test is constructed as

$$
S=n \hat{\psi}^{T} \hat{\psi}
$$

Under the null hypothesis, $S$ converges in distribution to the $\chi^{2}$ distribution with $K$ degrees of freedom as $n \rightarrow \infty$.

Compared to omnibus tests, smooth tests have attractive finite sample properties both in terms of size and power (see Rayner and Best (1990) for a comprehensive review on smooth tests). In addition, the smooth test has one particular appealing feature. In case the null hypothesis $\lambda=0$ is rejected, it is natural to consider $f_{K}$ as a plausible alternative density. In this sense, this test is not only diagnostic but also constructive for it provides useful pointers for subsequent analysis.

\subsection{Smooth tests of Copula Specifications: Simple Hypothesis}

To fix idea, we first present a smooth test of copula specification for the simplest case where the marginal distributions are known and the copula density in question is completely specified.

Let $X_{1}=\left(X_{11}, X_{21}\right)^{T}, \ldots, X_{n}=\left(X_{1 n}, X_{2 n}\right)^{T}$ be a random sample from a bivariate distribution function $F\left(x_{1}, x_{2}\right)$ with continuous marginal distributions $F_{1}\left(x_{1}\right), F_{2}\left(x_{2}\right)$. According to the theorem of Sklar (1959), there exists a unique copula $C$ such that

$$
F\left(x_{1}, x_{2}\right)=C\left(F_{1}\left(x_{1}\right), F_{2}\left(x_{2}\right)\right)
$$

for all $x_{1}, x_{2} \in \mathbb{R}$. Suppose $C$ is differentiable, there exists a corresponding copula density $c\left(v_{1}, v_{2}\right) \equiv$ $\partial^{2} / \partial v_{1} \partial v_{2} C\left(v_{1}, v_{2}\right)$.

Let $c^{0}\left(v_{1}, v_{2} ; \alpha\right)$, where $\left(v_{1}, v_{2}\right) \in[0,1]^{2}$, be a class of parametric copula density functions characterized by a finite $p$-dimensional parameter $\alpha$. We are interested in the following simple hypothesis

$$
H_{0}: \operatorname{Pr}\left(c\left(v_{1}, v_{2}\right)=c^{0}\left(v_{1}, v_{2} ; \alpha_{0}\right)\right)=1
$$

against the alternative hypothesis

$$
H_{1}: \operatorname{Pr}\left(c\left(v_{1}, v_{2}\right)=c^{0}\left(v_{1}, v_{2} ; \alpha_{0}\right)\right)<1
$$


for some $\alpha_{0} \in \mathcal{A}$, a compact subset of $\mathbb{R}^{p}$.

Let $g=\left(g_{1}, \ldots, g_{K}\right)^{T}$ be a series of linearly dependent bounded real valued functions defined on $[0,1]^{2}$. Define $\mu(g ; \alpha)=\int g\left(v_{1}, v_{2}\right) c^{0}\left(v_{1}, v_{2} ; \alpha\right) d v$ and

$$
\mathfrak{g}\left(v_{1}, v_{2} ; \alpha\right)=g\left(v_{1}, v_{2}\right)-\mu(g ; \alpha) .
$$

Define $U_{t}=\left(U_{1 t}, U_{2 t}\right)^{T}$ with $U_{j t}=F_{j}\left(X_{j t}\right), j=1,2$. Let

$$
\hat{\mathfrak{g}}\left(\alpha_{0}\right)=\frac{1}{n} \sum_{t=1}^{n} \mathfrak{g}\left(U_{1 t}, U_{2 t} ; \alpha_{0}\right) .
$$

and

$$
V\left(\alpha_{0}\right)=\operatorname{var}\left[\mathfrak{g}\left(U_{1 t}, U_{2 t} ; \alpha_{0}\right)\right] .
$$

It follows that under the null, $\hat{\mathfrak{g}}\left(\alpha_{0}\right) \stackrel{p}{\rightarrow} 0$ and $\sqrt{n} \hat{\mathfrak{g}}\left(\alpha_{0}\right) \stackrel{d}{\rightarrow} \mathcal{N}(0, V)$ as $n \rightarrow \infty$ under suitable regularity conditions.

Next let $\hat{V}\left(\alpha_{0}\right)=\frac{1}{n} \sum_{t=1}^{n} \mathfrak{g}\left(U_{1 t}, U_{2 t} ; \alpha_{0}\right) \mathfrak{g}\left(U_{1 t}, U_{2 t} ; \alpha_{0}\right)^{T}$. We construct a smooth test for copula specification as follows:

$$
Q \equiv n \hat{\mathfrak{g}}^{T}\left(\alpha_{0}\right)\left[\hat{V}\left(\alpha_{0}\right)\right]^{-1} \hat{\mathfrak{g}}\left(\alpha_{0}\right),
$$

which can be shown to converge in distribution to the $\chi^{2}$ distribution with $K$ degrees of freedom under suitable regularity conditions given in the next section. Compared with Neyman's smooth test of uniformity, the additional normalizing factor in the form of $V^{-1}$ is necessary since $\mathfrak{g}=$ $\left(\mathfrak{g}_{1}, \ldots, \mathfrak{g}_{K}\right)^{T}$ is generally not orthonormal with respect to the null copula density $c^{0}$.

Similar to Neyman's smooth test of uniformity, our smooth test of copula specification can be motivated by a smooth alternative. Consider the following density

$$
c_{g}\left(v_{1}, v_{2} ; \lambda\right)=c^{0}\left(v_{1}, v_{2} ; \alpha_{0}\right) \exp \left(\sum_{k=1}^{K} \lambda_{k} \mathfrak{g}_{k}\left(v_{1}, v_{2} ; \alpha_{0}\right)-\lambda_{0}\right)
$$

where

$$
\lambda_{0}=\log \int c^{0}\left(v_{1}, v_{2} ; \alpha_{0}\right) \exp \left(\sum_{k=1}^{K} \lambda_{k} \mathfrak{g}_{k}\left(v_{1}, v_{2} ; \alpha_{0}\right)\right) d v_{1} d v_{2} .
$$

Under the assumption that $\left(v_{1}, v_{2}\right)$ are distributed according to a distribution in the family of $(6)$, hypothesis (2) is equivalent to that $\lambda=0$. It is seen that (5) is a score test on this hypothesis.

We note that (6) can be derived as the density that minimizes the Kullback-Leibler information criterion between the target density and the null density subject to side moment conditions associated with $g$ (cf. Efron and Tibshirani (1996)). In particular, consider the following optimization problem which minimizes the Kullback-Leibler information criterion between the null copula 
density $c^{0}$ and a generic density $c:[0,1]^{2} \rightarrow \mathbb{R}$

$$
\min _{c} \int c\left(v_{1}, v_{2}\right) \log \frac{c\left(v_{1}, v_{2}\right)}{c^{0}\left(v_{1}, v_{2} ; \alpha_{0}\right)} d v_{1} d v_{2}
$$

subject to

$$
\begin{aligned}
& \int c\left(v_{1}, v_{2}\right) d v_{1} d v_{2}=1, \\
& \int \mathfrak{g}_{k}\left(v_{1}, v_{2} ; \alpha_{0}\right) c\left(v_{1}, v_{2}\right) d v_{1} d v_{2}=\hat{\mathfrak{g}}_{k}, k=1, \ldots, K .
\end{aligned}
$$

The solution takes the form

$$
c_{g}\left(v_{1}, v_{2} ; \hat{\lambda}\right)=c^{0}\left(v_{1}, v_{2} ; \alpha_{0}\right) \exp \left(\sum_{k=1}^{K} \hat{\lambda}_{k} \mathfrak{g}_{k}\left(v_{1}, v_{2} ; \alpha_{0}\right)-\hat{\lambda}_{0}\right),
$$

where $\hat{\lambda}=\left(\hat{\lambda}_{1}, \ldots, \hat{\lambda}_{K}\right)^{T}$ are Lagrangian multipliers associated with the side moment conditions (7). Therefore, if the hypothesis $\lambda=0$ is rejected by (5), it is reasonable to consider (8) as an alternative density, which has the appealing interpretation that among all densities satisfying the sample moment conditions (7), it is the unique density within the family of (6) that is closest to the null copula density in terms of the Kullback-Leibler information criterion.

\section{Semiparametric Smooth Copula Tests}

In practice, the marginal distributions and copula parameters are usually unknown and therefore have to be estimated. Consequently, the hypothesis of interest becomes a composite one as follows:

$$
H_{0}: \operatorname{Pr}\left(c\left(v_{1}, v_{2}\right)=c^{0}\left(v_{1}, v_{2} ; \alpha_{0}\right)\right)=1 \text { for some } \alpha_{0} \in \mathcal{A}
$$

against the alternative hypothesis

$$
H_{1}: \operatorname{Pr}\left(c\left(v_{1}, v_{2}\right)=c^{0}\left(v_{1}, v_{2} ; \alpha\right)\right)<1 \text { for all } \alpha \in \mathcal{A}
$$

One can estimate the marginal distributions using parametric or nonparametric methods. Chen (2007) discusses moment-based tests using parametrically estimated marginal distributions. Although efficient under correct parametric specification, the test can suffer size distortion and power loss under incorrect distributional assumptions. In this study, we focus on copula specification tests where the marginal distributions are estimated nonparametrically by their empirical distributions. Compared with parametric estimates, the empirical CDF's are consistent. On the other hand, compared with nonparametric estimates such as kernel distribution estimates, the empirical CDF's are free of smoothing parameters. 
Lacking a priori guidance on the marginal distributions, we estimate $F_{j}$ by the rescaled empirical distribution function,

$$
\tilde{F}_{j}(x)=\frac{1}{n+1} \sum_{t=1}^{n} 1\left(X_{j t} \leq x\right), j=1,2,
$$

where $1(\cdot)$ is the indicator function. Let $\tilde{U}_{t} \equiv\left(\tilde{U}_{1 t}, \tilde{U}_{2 t}\right)^{T}$ with $\tilde{U}_{j t}=\tilde{F}_{j}\left(X_{j t}\right), j=1,2$. We proceed to estimate copula parameters via the maximum likelihood estimation as follows:

$$
\hat{\alpha}=\arg \max _{\alpha}\left[\frac{1}{n} \sum_{t=1}^{n} \log c^{0}\left\{\tilde{U}_{1 t}, \tilde{U}_{2 t} ; \alpha\right\}\right] .
$$

One can envision that a smooth test on the hypothesis (9) can be constructed based on

$$
\hat{\mathfrak{g}}(\hat{\alpha}) \equiv \frac{1}{n} \sum_{t=1}^{n} \mathfrak{g}\left(\tilde{U}_{1 t}, \tilde{U}_{2 t} ; \hat{\alpha}\right)
$$

which is analogous to (3) with $U_{j t}$ replaced by $\tilde{U}_{j t}, j=1,2$ and $\alpha_{0}$ by $\hat{\alpha}$. However the test (5) for simple hypotheses is not directly applicable here because of the presence of nuisance parameters in (11). There are two sets of nuisance parameters: the finite dimensional copula parameter $\alpha$ and the marginal distributions $F_{j}, j=1,2$, which are infinite-dimensional when estimated nonparametrically.

In order to construct a test based on (12), we need to account for the influences of the nuisance parameters. We start with the asymptotic distribution of $\hat{\alpha}$, which is studied by Genest et al. (1995) and Chen and Fan (2005). Let $l\left(v_{1}, v_{2} ; \alpha\right)=\log c\left(v_{1}, v_{2} ; \alpha\right)$ and use indices $\alpha$ and $j=1,2$ to denote partial derivatives of $l$ with respect to $\alpha$ and $v_{j}, j=1,2$ respectively. Also define

$$
W_{j}\left(U_{j t} ; \alpha\right) \equiv \mathrm{E}\left[l_{\alpha j}\left(U_{1 s}, U_{2 s} ; \alpha\right)\left\{1\left(U_{j t} \leq U_{j s}\right)-U_{j s}\right\} \mid U_{j t}\right]
$$

We can then show the following:

Theorem 1. Suppose $\mathrm{E}\left[l\left(U_{1 t}, U_{2 t} ; \alpha\right)\right]$ has a unique maximum at $\alpha_{0}$. Under the regularity conditions given in the Appendix, the semiparametric estimator $\hat{\alpha} \stackrel{p}{\rightarrow} \alpha_{0}$ and $\sqrt{n}\left(\hat{\alpha}-\alpha_{0}\right) \stackrel{d}{\rightarrow} \mathcal{N}\left(0, B^{-1} \Sigma B^{-1}\right)$, where

$$
\begin{aligned}
& B \equiv-\mathrm{E}\left[l_{\alpha \alpha}\left(U_{1 t}, U_{2 t} ; \alpha_{0}\right)\right], \\
& \Sigma \equiv \operatorname{var}\left[l_{\alpha}\left(U_{1 t}, U_{2 t} ; \alpha_{0}\right)+\sum_{j=1}^{2} W_{j}\left(U_{j t} ; \alpha_{0}\right)\right] .
\end{aligned}
$$

with $l_{\alpha \alpha}$ being the second derivative of $l$ with respect to $\alpha$. 
Under the null, we have $B=\mathrm{E}\left[l_{\alpha}\left(U_{1 t}, U_{2 t} ; \alpha_{0}\right) l_{\alpha}\left(U_{1 t}, U_{2 t} ; \alpha_{0}\right)^{T}\right]$ by the information matrix equality. In addition, since the conditional expectation of $l_{\alpha}\left(U_{1 t}, U_{2 t} ; \alpha_{0}\right)$ with respect to either $U_{j t}$ is zero, $l_{\alpha}\left(U_{1 t}, U_{2 t} ; \alpha_{0}\right)$ is uncorrelated with each $W_{j}\left(U_{j t} ; \alpha_{0}\right)$. Therefore, the asymptotic variance of $\hat{\alpha}$ can be simplified to

$$
B^{-1}+B^{-1} \operatorname{var}\left[\sum_{j=1}^{2} W_{j}\left(U_{j t} ; \alpha_{0}\right)\right] B^{-1}
$$

Obviously the asymptotic variance of $\hat{\alpha}$ is reduced to $B^{-1}$ when the marginal distributions are known.

Next let

$$
\begin{aligned}
& \hat{B}=\frac{1}{n} \sum_{t=1}^{n} l_{\alpha}\left(\tilde{U}_{1 t}, \tilde{U}_{2 t} ; \hat{\alpha}\right) l_{\alpha}\left(\tilde{U}_{1 t}, \tilde{U}_{2 t} ; \hat{\alpha}\right)^{T}, \\
& \hat{W}_{j t}=\frac{1}{n} \sum_{s=1, s \neq t}^{n} l_{\alpha j}\left(\tilde{U}_{1 s}, \tilde{U}_{2 s} ; \hat{\alpha}\right)\left\{1\left(\tilde{U}_{j t} \leq \tilde{U}_{j s}\right)-\tilde{U}_{j s}\right\} .
\end{aligned}
$$

The asymptotic variance of $\hat{\alpha}$ can be consistently estimated by

$$
\hat{B}^{-}+\hat{B}^{-}\left\{\frac{1}{n} \sum_{t=1}^{n}\left(\sum_{j=1}^{2} \hat{W}_{j t}\right)\left(\sum_{j=1}^{2} \hat{W}_{j t}\right)^{T}\right\} \hat{B}^{-}
$$

where $\hat{B}^{-}$is the generalized inverse of $\hat{B}$.

Let $g_{(j)}\left(v_{1}, v_{2}\right)=\partial g\left(v_{1}, v_{2}\right) / \partial v_{j}$. Further define

$$
\begin{aligned}
G & \equiv \frac{\partial}{\partial \alpha} \mu\left(g ; \alpha_{0}\right), \\
Z_{j}\left(U_{j t}\right) & \equiv \mathrm{E}\left[g_{(j)}\left(U_{1 s}, U_{2 s}\right)\left\{1\left(U_{j t} \leq U_{j s}\right)-U_{j s}\right\} \mid U_{j t}\right] .
\end{aligned}
$$

We obtain the following asymptotic distribution for $\hat{\mathfrak{g}}(\hat{\alpha})$.

Theorem 2. Suppose the regularity conditions given in the Appendix hold. Under the null hypothesis, $\hat{\mathfrak{g}}(\hat{\alpha}) \stackrel{p}{\rightarrow} 0$ and $\sqrt{n} \hat{\mathfrak{g}}(\hat{\alpha}) \stackrel{d}{\rightarrow} \mathcal{N}(0, \Omega)$, where

$$
\Omega=\operatorname{var}\left[\mathfrak{g}\left(U_{1 t}, U_{2 t} ; \alpha_{0}\right)+\sum_{j=1}^{2} Z_{j}\left(U_{j t}\right)+G^{T} B^{-1}\left\{l_{\alpha}\left(U_{1 t}, U_{2 t} ; \alpha_{0}\right)+\sum_{j=1}^{2} W_{j}\left(U_{j t} ; \alpha_{0}\right)\right\}\right] .
$$

Remark 1. The asymptotic variance $\Omega$ reflects the influence of nuisance parameters in $\hat{\mathfrak{g}}(\hat{\alpha})$. If the marginal distributions are known, the terms involving $W$ 's and $Z$ 's can be dropped, resulting in

$$
\Omega=\operatorname{var}\left[\mathfrak{g}\left(U_{1 t}, U_{2 t} ; \alpha_{0}\right)+G^{T} B^{-1}\left\{l_{\alpha}\left(U_{1 t}, U_{2 t} ; \alpha_{0}\right)\right\}\right]
$$


We note that this simplified variance can be constructed alternatively according to

$$
\begin{aligned}
\Omega^{-1} & =\left(V-G^{T} B^{-1} G\right)^{-1} \\
& =V^{-1}+V^{-1} G^{T}\left(B-G V^{-1} G^{T}\right)^{-1} G V^{-1},
\end{aligned}
$$

where $V \equiv \operatorname{var}\left[\mathfrak{g}\left(U_{1 t}, U_{2 t} ; \alpha_{0}\right)\right]$ as is given in (4). This result can be obtained via a standard modification of quadratic score statistic (cf. Thomas and Pierce (1979)).

Remark 2. When the copula parameter $\alpha$ is known, the third term on the right hand side of (15) can be dropped. When both the marginal distributions and copula parameter are known, $\Omega$ is reduced to $\operatorname{var}\left[\mathfrak{g}\left(U_{1 t}, U_{2 t} ; \alpha_{0}\right)\right]$, which is exactly the variance (4) derived in the previous section. Another possible incidence of $\Omega=V$ is when the true copula approaches the independence copula, a result readily available from Proposition 2.2 of Genest et al. (1995).

Remark 3. Since the conditional expectations of $\mathfrak{g}\left(U_{1 t}, U_{2 t} ; \alpha_{0}\right)$ and of $l_{\alpha}\left(U_{1 t}, U_{2 t} ; \alpha_{0}\right)$ with respect to either $U_{j t}$ are zeros under the null hypothesis, these two terms are uncorrelated with each $W_{j}\left(U_{j t} ; \alpha_{0}\right)$ or $Z_{j}\left(U_{j t} ; \alpha_{0}\right)$. Therefore, the asymptotic variance of $\hat{\alpha}(\hat{\theta})$ can be simplified to

$$
\begin{aligned}
\Omega= & \operatorname{var}\left[\mathfrak{g}\left(U_{1 t}, U_{2 t} ; \alpha_{0}\right)+G^{T} B^{-1} l_{\alpha}\left(U_{1 t}, U_{2 t} ; \alpha_{0}\right)\right] \\
& +\operatorname{var}\left[\sum_{j=1}^{2} Z_{j}\left(U_{j t}\right)+G^{T} B^{-1}\left\{\sum_{j=1}^{2} W_{j}\left(U_{j t} ; \alpha_{0}\right)\right\}\right] .
\end{aligned}
$$

Next we present a consistent estimator for $\Omega$. Let

$$
\varphi_{t}=\mathfrak{g}\left(\tilde{U}_{1 t}, \tilde{U}_{2 t} ; \hat{\alpha}\right)+\sum_{j=1}^{2} \hat{Z}_{j t}+\hat{G}^{T} \hat{B}^{-}\left(l_{\alpha}\left(\tilde{U}_{1 t}, \tilde{U}_{2 t} ; \hat{\alpha}\right)+\sum_{j=1}^{2} \hat{W}_{j t}\right)
$$

where

$$
\begin{aligned}
\hat{G} & =\frac{\partial}{\partial \alpha} \mu(g ; \hat{\alpha}) \\
\hat{Z}_{j t} & =\frac{1}{n} \sum_{s=1, s \neq t}^{n} g_{(j)}\left(\tilde{U}_{1 s}, \tilde{U}_{2 s}\right)\left\{1\left(\tilde{U}_{j t} \leq \tilde{U}_{j s}\right)-\tilde{U}_{j s}\right\} .
\end{aligned}
$$

It follows that $\Omega$ can be estimated consistently by

$$
\hat{\Omega}=\frac{1}{n} \sum_{t=1}^{n} \varphi_{t} \varphi_{t}^{T}
$$

Alternatively we can estimate $\Omega$ using the sample analog of (16).

We can now construct a smooth test of copula specification as follows. 
Theorem 3. Under the regularity conditions given in the Appendix, the semiparametric smooth test of copula specification is given by

$$
\hat{Q} \equiv n \hat{\mathfrak{g}}(\hat{\alpha})^{T} \hat{\Omega}^{-1} \hat{\mathfrak{g}}(\hat{\alpha})
$$

Under the null hypothesis, $\hat{Q} \stackrel{d}{\rightarrow} \chi_{K}^{2}$ as $n \rightarrow \infty$.

Similar to the results on simple hypotheses, when the composite null hypothesis is rejected by test (19), we can construct an alternative copula density according to (8), which provides a plausible approximation to the unknown copula density.

\section{Extension to copula-based multivariate dynamic models}

So far we have maintained that our samples are iid realizations of random distributions. One area that copula methods have found most applications is financial economics, wherein the iid assumption usually does not hold. In this section, we extend the proposed smooth test to the semiparametric copula-based multivariate dynamic (SCOMDY) models studied by Chen and Fan (2006) and Chan et al. (2009). Following Chen and Fan (2006), we consider a class of SCOMDY models for a bivariate time series $X_{t}=\left(X_{1 t}, X_{2 t}\right), t=1, \ldots, T$. Denote by $\mathcal{F}_{t-1}$ the information set that contains information from the past and from exogenous variables. The model is given by

$$
X_{t}=\mu_{t}(\theta)+\sqrt{H_{t}(\theta)} \varepsilon_{t},
$$

where

$$
\mu_{t}(\theta)=\left(\mu_{1 t}(\theta), \mu_{2 t}(\theta)\right)^{\prime}=E\left[X_{t} \mid \mathcal{F}_{t-1}\right]
$$

is the conditional mean of $X_{t}$ given $\mathcal{F}_{t-1}$, and

$$
H_{t}(\theta)=\operatorname{diag}\left(h_{1 t}(\theta), h_{2 t}(\theta)\right)
$$

in which

$$
h_{j t}(\theta)=E\left[\left(X_{j t}-\mu_{j t}(\theta)\right)^{2} \mid \mathcal{F}_{t-1}\right], j=1,2,
$$

is the conditional variance of $X_{j t}$ given $\mathcal{F}_{t-1}$. We assume that the model is correctly parameterized up to a finite-dimensional unknown parameter $\theta$. Moreover, $\varepsilon_{t}=\left(\varepsilon_{1 t}, \varepsilon_{2 t}\right)^{T}$ is a sequence of iid random vectors with a distribution function $F(\varepsilon)=C\left(F_{1}\left(\varepsilon_{1}\right), F_{2}\left(\varepsilon_{2}\right) ; \alpha\right)$, where $F_{j}(\cdot)$ is the true but unknown continuous marginal distribution of $\varepsilon_{j t}$.

Chen and Fan (2006) proposed a three-step estimator $\hat{\alpha}$ of the copula parameter $\alpha_{0}$. In the first step, the dynamic parameters are estimated consistently by $\hat{\theta}$, usually the MLE. In the second step, the distributions of the standardized error terms of the two marginal distributions are estimated by their empirical distributions. Denote by $\tilde{U}_{j t}(\hat{\theta}), j=1,2$ the empirical CDF's thus obtained. In the 
third step, the copula parameter $\hat{\alpha}(\hat{\theta})$ are estimated using the MLE based on $\left(\tilde{U}_{1 t}(\hat{\theta}), \tilde{U}_{2 t}(\hat{\theta})\right), t=$ $1, \ldots, T$.

Replacing $\hat{\alpha}$ in equation (12) with $\hat{\alpha}(\hat{\theta})$, we have under the SCOMDY framework

$$
\hat{\mathfrak{g}}(\hat{\alpha}, \hat{\theta})=\frac{1}{T} \sum_{t=1}^{T} g\left(\tilde{U}_{1 t}(\hat{\theta}), \tilde{U}_{2 t}(\hat{\theta})\right)-\mu(g ; \hat{\alpha}(\hat{\theta})) .
$$

An important contribution of Chen and Fan (2006) is the finding that asymptotic distribution of $\hat{\alpha}(\hat{\theta})$ is not affected by the estimation of dynamic parameters when the marginal distributions are estimated by the empirical CDF's. Thanks to the invariance result, the asymptotic distribution of $\hat{\mathfrak{g}}(\hat{\alpha}, \hat{\theta})$ is readily available.

Theorem 4. Suppose the regularity conditions given in the Appendix hold. Under the null hypothesis that $C\left(F_{1}\left(\epsilon_{1}\right), F_{2}\left(\epsilon_{2}\right)\right)=C_{0}\left(U_{1}, U_{2} ; \alpha_{0}\right), \hat{\mathfrak{g}}(\hat{\alpha}, \hat{\theta}) \stackrel{p}{\rightarrow} 0$ and $\sqrt{T} \hat{\mathfrak{g}}(\hat{\alpha}, \hat{\theta}) \stackrel{d}{\rightarrow} \mathcal{N}(0, \Omega)$, where $\Omega$ is defined in equation (15).

It follows immediately that one can construct a smooth test of copula specification for the SCOMDY in a similar manner.

Theorem 5. Suppose the regularity conditions given in the Appendix hold. The semiparametric smooth test of copula specification is given by

$$
\hat{Q} \equiv T \hat{\mathfrak{g}}(\hat{\alpha}, \hat{\theta})^{T} \hat{\Omega}^{-1} \hat{\mathfrak{g}}(\hat{\alpha}, \hat{\theta})
$$

where $\hat{\Omega}$ is defined as (18) with $\tilde{U}_{1 i}, \tilde{U}_{2 i}$ and $\hat{\alpha}$ replaced by $\tilde{U}_{1 i}(\hat{\theta}), \tilde{U}_{2 i}(\hat{\theta})$ and $\hat{\alpha}(\hat{\theta})$. Under the null hypothesis, $\hat{Q} \stackrel{d}{\rightarrow} \chi_{K}^{2}$ as $n \rightarrow \infty$.

Remark 4. The appealing property that the asymptotic distribution of $\hat{\mathfrak{g}}(\hat{\alpha}, \hat{\theta})$ is not affected by the estimation of dynamic parameters in the SCOMDY models only holds when the marginal distributions are estimated by the empirical CDF's and the copula in question is time invariant (Rémillard, 2010).

\section{Monte Carlo Simulations}

We conduct a series of Monte Carlo simulations to assess the finite-sample performance of the proposed tests. Since the Gaussian copula is the most commonly used copula in practice, we focus on the Gaussian copula as the null copula distribution. We generate the marginal distributions from the standard normal distributions and consider six different copula distributions, falling into three categories:

(1) Radially symmetric copulas: ${ }^{1}$ the Gaussian copula $\left(C_{N}\right)$, the Student's $t$ copula with four degrees of freedom $\left(C_{t_{4}}\right)$, and Plackett copula $\left(C_{P}\right)$. The Gaussian copula has zero tail dependence

\footnotetext{
${ }^{1}$ A copula $C$ is radially symmetric if $C\left(1-v_{1}, 1-v_{2}\right)=1-v_{1}-v_{2}+C\left(v_{1}, v_{2}\right)$ for all $\left(v_{1}, v_{2}\right) \in[0,1]^{2}$.
} 
while the Student's $t$ copula exhibits symmetric tail dependence. The Plackett copula is defined as

$$
C_{P}\left(v_{1}, v_{2}, \alpha\right)= \begin{cases}\left(\left[1+(\alpha-1)\left(v_{1}+v_{2}\right)\right]-\sqrt{\left[1+(\alpha-1)\left(v_{1}+v_{2}\right)\right]^{2}-4 v_{1} v_{2} \alpha(\alpha-1)}\right) /[2(\alpha-1)] & \text { for } \alpha>0, \alpha \neq 1, \\ v_{1} v_{2} & \text { for } \alpha=1 .\end{cases}
$$

The Plackett copula is comprehensive in the following sense: As $\alpha$ goes to 0 or $\infty$, the copula approaches the lower or upper Fréchet-Hoeffding bound; when $\alpha=1$, it becomes the independent copula. The Plackett copula has been widely used as an alternative to the bivariate Gaussian copula for studies of power and robustness of various statistical tests (cf. Chen and Fan, 2006; Genest et al., 2009).

(2) Symmetric but not radially symmetric copulas: ${ }^{2}$ Clayton copula $\left(C_{C}\right)$ and Gumbel-Hougaard copula $\left(C_{G}\right)$. In contrast to radially symmetric copulas, the Clayton copula exhibits strong left tail dependence and relatively weak right tail dependence. On the other hand, the Gumbel-Hougaard copula exhibits strong right tail dependence and relatively weak left tail dependence.

(3) Asymmetric copulas: asymmetric Gumbel-Hougaard copula $\left(C_{A G}\right)$. The asymmetric GumbelHougaard copula is transformed from the symmetric Gumbel-Hougaard copula using Khoudraji's device (Khoudraji, 1995) as follows,

$$
C_{A G}\left(v_{1}, v_{2} ; \lambda, k, \alpha\right)=v_{1}^{1-\lambda} v_{2}^{1-k} C_{G}\left(v_{1}^{\lambda}, v_{2}^{k} ; \alpha\right)
$$

for all $v_{1}, v_{2} \in[0,1]$ and arbitrary choices of $\lambda \neq k \in(0,1)$.

For each copula, we consider three scenarios with the correlation coefficient $\rho=0.2,0.5$ and 0.8 , corresponding to a low, median and high degree of dependence. The parameters for non-Gaussian copulas are calculated by inversion of Kendall's tau, which is defined as,

$$
\tau=4 \int C\left(v_{1}, v_{2}\right) d C\left(v_{1}, v_{2}\right)-1
$$

The values of $\tau$ corresponding to $\rho=0.2,0.5$ and 0.8 are $0.1282,0.3317$ and 0.5903 . For a detailed account of the various copulas considered in this study and their estimations, see Nelsen (1993). We run simulations with sample size $n=500,1000$ and each experiment is repeated 1,000 times.

One appealing feature of the proposed smooth test is its flexibility such that one can tailor the moment functions, $g\left(v_{1}, v_{2}\right)$, according to his research needs. Several general considerations apply here. First, the functions shall be linearly independent. Second, as is well known about Neyman's smooth test, its asymptotic power suffers when the degree of freedom, $K$, is large. Usually, a small number of terms is used. For instance, Neyman (1937) recommends $K \leq 4$. Third, ideally one shall choose the moment functions that best capture deviations from the null distributions.

Taking all these factors into consideration, when there is no a priori reason to focus on a particular direction of deviation, we select the moment functions from the normalized Legendre

\footnotetext{
${ }^{2}$ A copula $C$ is symmetric if $C\left(v_{1}, v_{2}\right)=C\left(v_{2}, v_{1}\right)$ for all $\left(v_{1}, v_{2}\right) \in[0,1]^{2}$.
} 
polynomials. We focus on the first three Legendre polynomials given by, for $v \in[0,1]$,

$$
\begin{aligned}
& \psi_{1}(v)=\sqrt{3}(2 v-1) \\
& \psi_{2}(v)=\sqrt{5}\left(6 v^{2}-6 v+1\right), \\
& \psi_{3}(v)=\sqrt{7}\left(20 v^{3}-30 v^{2}+12 v-1\right) .
\end{aligned}
$$

Our $g$ functions then consist of various tensor products of these basis functions under the restriction that the maximum number of terms is 4 . Table 1 summarizes the various configurations considered in this study.

Table 1: Configurations of $g\left(v_{1}, v_{2}\right)$

\begin{tabular}{llc}
\hline Group & $g\left(v_{1}, v_{2}\right)$ & Notation \\
\hline & $g_{1}=\psi_{1} \psi_{1}$ & $g_{S_{1}}$ \\
& $g_{1}=\psi_{2} \psi_{2}$ & $g_{S_{2}}$ \\
Singleton & $g_{1}=\psi_{3} \psi_{3}$ & $g_{S_{3}}$ \\
& $g_{1}=\psi_{1} \psi_{2}$ & $g_{S_{4}}$ \\
& $g_{1}=\psi_{2} \psi_{1}$ & $g_{S_{5}}$ \\
& $g_{1}=\psi_{1} \psi_{3}$ & $g_{S_{6}}$ \\
& $g_{1}=\psi_{3} \psi_{1}$ & $g_{S_{7}}$ \\
\hline \multirow{5}{*}{ Diagonal } & $g_{D_{1}}$ \\
& $g_{1}=\psi_{1} \psi_{1}, g_{2}=\psi_{2} \psi_{2}$ & $g_{D_{2}}$ \\
& $g_{1}=\psi_{1} \psi_{1}, g_{2}=\psi_{2} \psi_{2}, g_{3}=\psi_{3} \psi_{3}$ & $g_{D_{3}}$ \\
& $g_{1}=\psi_{1} \psi_{2}, g_{2}=\psi_{2} \psi_{1}$ & $g_{D_{4}}$ \\
\hline & $g_{1}=\psi_{1} \psi_{3}, g_{2}=\psi_{2} \psi_{2}, g_{3}=\psi_{3} \psi_{1}$ & $g_{O_{1}}$ \\
Others & $g_{1}=\psi_{1} \psi_{1}, g_{2}=\psi_{1} \psi_{2}, g_{3}=\psi_{2} \psi_{2}$ & $g_{O_{2}}$ \\
& $g_{1}=\psi_{1} \psi_{2}, g_{2}=\psi_{2} \psi_{2}, g_{3}=\psi_{2} \psi_{1}$ & $g_{O_{3}}$ \\
& $g_{1}=\psi_{1} \psi_{1}, g_{2}=\psi_{1} \psi_{2}, g_{3}=\psi_{2} \psi_{1}, g_{4}=\psi_{2} \psi_{2}$ & $g_{O_{4}}$ \\
\hline & $g_{1}=\psi_{1} \psi_{1}, g_{2}=\psi_{1} \psi_{3}, g_{3}=\psi_{2} \psi_{2}, g_{4}=\psi_{3} \psi_{3}$ & \\
\hline
\end{tabular}

To save place, we use $g_{1}=\psi_{1} \psi_{1}$ to denote the function $g_{1}\left(v_{1}, v_{2}\right)=\psi_{1}\left(v_{1}\right) \psi_{1}\left(v_{2}\right)$, and all other terms are defined similarly. A few explanations are in order for the configurations listed in Table 1. Consider the tensor product of $\left(\psi_{1}, \psi_{2}, \psi_{3}\right)$ as a 3 by 3 matrix with the $(i, j)$ th entry being $\psi_{i} \psi_{j}$. The first group 'Singleton' contains single entry located either on the diagonal or off-diagonal of the matrix. The group 'Diagonal' contains multiple entries on the diagonal or anti-diagonal. The group 'Others' contains other configurations. $g_{O_{1}}$ and $g_{O_{2}}$ contain entries in the upper right and lower right triangular matrix with $i+j \leq 4 ; g_{O_{3}}$ contains all entries with $i, j \leq 2 ; g_{O_{4}}$ contains entries with $i+j$ being an even number and $1 \leq i, j \leq 3$.

In what follows, we refer to the smooth tests with moment functions $g_{S 1}$ as $Q_{S 1}$ and all other tests are defined similarly. We report the empirical sizes and powers of the tests in Table 2 . The columns under the header $C_{N}$ correspond to the test results with the Gaussian copula as the true copula function and reflect the empirical size. The rest of the table reflects powers of the test 
against various alternatives. Overall, the tests exhibit correct sizes, centering about the nominal value of $5 \%$. Generally both size and power improve with sample size. We also note that the test performance varies across the configurations of moment functions, the types of true copula families and the degree of dependence. A closer examination of the results offers the following interesting insights.

(1) For tests within the elliptical copula family, since the distributions share many common features, the tests seemed to be driven by one or two moments. When the alternative distribution is the Student's $t$ copula, the term $\psi_{2} \psi_{2}$ contributes significantly to the power, reflecting the fattailedness of $t$-copulas. The term $\psi_{3} \psi_{3}$ also has non-trivial power, which increases with the degree of dependence substantially. As a result, all tests including either or both of these two terms register good powers against the $t$-copula. As for the Plackett copula, the term $\psi_{1} \psi_{1}$ contributes most discriminant power, while $\psi_{1} \psi_{3}$ and $\psi_{3} \psi_{1}$, followed by $\psi_{2} \psi_{2}$, also show good discriminant powers as the degree of dependence increases.

Since this family of copula distributions is radially symmetric, terms such as $\psi_{1} \psi_{2}$ and $\psi_{2} \psi_{1}$ have zero expectations and therefore do not have discriminant powers against distributions in the same family. This conjecture is confirmed by our experiments: tests with a single moment function $\psi_{1} \psi_{2}$ or $\psi_{2} \psi_{1}$ are shown to have powers close to the nominal size.

(2) In contrast to tests against elliptical families, the tests against copulas that are symmetric but not radially symmetric owe their most significant powers to off-diagonal terms. When the alternative distributions are Clayton or Gumbel copulas, terms like $\psi_{1} \psi_{2}$ and $\psi_{2} \psi_{1}$ are shown to have consistently high discriminant powers. Consequently, tests including these terms have outstanding power performance.

(3) As for the asymmetric Gumble-Hougaard copula, the discriminant power of various terms depends on the degree of dependence; no clear pattern is discerned. It is noteworthy that tests including the term $\psi_{1} \psi_{2}$ are consistently powerful across different degrees of dependence, even with the small sample size at $n=500$.

(4)The results also suggest that tests with multiple instruments outperform those with a single instrument in most cases. This is important because in many practical cases, researchers have no a priori guidance on how to select a particular direction for specification test. It is encouraging that a combination of a small number of terms appears to be a safe testing strategy that delivers satisfactory performance.

Next we report simulations on SCOMDY models as in Chen and Fan (2006). Following Chen et al. (2004), we consider the following dynamic data generating process (DGP)

$$
\begin{aligned}
X_{j t} & =0.01+0.05 X_{j, t-1}+e_{j t} \\
e_{j t} & =\sqrt{h_{j t}} \cdot \Phi^{-1}\left(U_{j t}\right) \\
h_{j t} & =0.05+0.85 h_{j, t-1}+0.1 e_{j t-1}^{2}, j=1,2
\end{aligned}
$$


Table 2: Empirical sizes and powers of the constructed tests (\%)

\begin{tabular}{|c|c|c|c|c|c|c|c|c|c|c|c|c|c|}
\hline \multirow[b]{2}{*}{$\rho$} & \multirow[b]{2}{*}{ Test } & \multicolumn{2}{|c|}{$C_{N}$} & \multicolumn{2}{|c|}{$C_{t_{4}}$} & \multicolumn{2}{|c|}{$C_{P}$} & \multicolumn{2}{|c|}{$C_{C}$} & \multicolumn{2}{|c|}{$C_{G}$} & \multicolumn{2}{|c|}{$C_{A G}$} \\
\hline & & $\mathrm{n}=500$ & 1000 & $\mathrm{n}=500$ & 1000 & $\mathrm{n}=500$ & 1000 & $\mathrm{n}=500$ & 1000 & $\mathrm{n}=500$ & 1000 & $\mathrm{n}=500$ & 1000 \\
\hline \multirow[t]{15}{*}{0.2} & $Q_{S_{1}}$ & 4.4 & 4.6 & 3.3 & 3.7 & 17.8 & 40 & 5 & 5.8 & 6.2 & 7.9 & 57.5 & 92.1 \\
\hline & $Q_{S_{2}}$ & 5.1 & 5.9 & 96.6 & 100 & 5 & 4.9 & 9.7 & 15.1 & 17.4 & 26.6 & 50.3 & 78.9 \\
\hline & $Q_{S_{3}}$ & 5 & 4.4 & 18.3 & 34.6 & 3.3 & 4.6 & 7.6 & 12.4 & 15.9 & 17.3 & 25.9 & 46.9 \\
\hline & $Q_{S_{4}}$ & 5.5 & 5.4 & 6.1 & 5.2 & 5.3 & 4.6 & 42 & 71.8 & 20.8 & 36.4 & 92.6 & 100 \\
\hline & $Q_{S_{5}}$ & 5.2 & 5.3 & 6.5 & 6.1 & 5.4 & 5.1 & 41.3 & 72.9 & 20.7 & 34.9 & 7.4 & 10.9 \\
\hline & $Q_{S_{6}}$ & 4.9 & 5.8 & 5.3 & 4.6 & 12.2 & 18.3 & 4.6 & 5.2 & 4.2 & 5.6 & 55.5 & 88.2 \\
\hline & $Q_{S_{7}}$ & 5.1 & 5.1 & 4.1 & 3.7 & 11.2 & 18.6 & 4.8 & 5.6 & 5.4 & 5.7 & 25.8 & 47.9 \\
\hline & $Q_{D_{1}}$ & 4.1 & 5.3 & 93.2 & 100 & 16.7 & 33.2 & 7.1 & 12.7 & 11.5 & 18.6 & 96.5 & 100 \\
\hline & $Q_{D_{2}}$ & 4.3 & 5.5 & 90 & 99.9 & 13.3 & 26.3 & 7.1 & 11 & 10.7 & 27.1 & 95 & 100 \\
\hline & $Q_{D_{3}}$ & 6 & 5.6 & 5.9 & 5 & 5.3 & 4.5 & 54 & 83.6 & 21.6 & 43.1 & 89.4 & 100 \\
\hline & $Q_{D_{4}}$ & 5.1 & 4.2 & 90 & 99.7 & 12.5 & 21.8 & 6.2 & 11.4 & 11 & 19 & 95.3 & 100 \\
\hline & $Q_{O_{1}}$ & 5.8 & 4.1 & 89.9 & 99.7 & 14.6 & 25.2 & 31.5 & 59.7 & 18 & 36 & 100 & 100 \\
\hline & $Q_{\mathrm{O}_{2}}$ & 5.5 & 5.6 & 89.9 & 99.7 & 6.1 & 4.9 & 50.2 & 83.6 & 27.4 & 49.6 & 96.7 & 100 \\
\hline & $Q_{O_{3}}$ & 5.8 & 5.8 & 85.6 & 98.9 & 13.2 & 23.5 & 45.1 & 76.4 & 20.9 & 46.2 & 100 & 100 \\
\hline & $Q_{O_{4}}$ & 4 & 4.3 & 85.9 & 99.8 & 10.9 & 21.8 & 7.4 & 10.5 & 9.2 & 18.9 & 96.1 & 99.9 \\
\hline \multirow[t]{15}{*}{0.5} & $Q_{S_{1}}$ & 4.2 & 4.5 & 3.1 & 4.3 & 62.5 & 94.2 & 0.8 & 1.6 & 3.3 & 5.4 & 7.1 & 15.5 \\
\hline & $Q_{S_{2}}$ & 5.2 & 4.5 & 92.6 & 99.8 & 12.4 & 23.6 & 13.8 & 24.1 & 24.6 & 51.2 & 2.9 & 3 \\
\hline & $Q_{S_{3}}$ & 4.5 & 4.2 & 70.1 & 94.5 & 5.9 & 11 & 33.1 & 64 & 32.3 & 58 & 3.7 & 6.7 \\
\hline & $Q_{S_{4}}$ & 4.4 & 4.4 & 5.5 & 4.6 & 4.4 & 5.5 & 99 & 100 & 61 & 89.2 & 100 & 100 \\
\hline & $Q_{S_{5}}$ & 4.7 & 5.4 & 5.5 & 3.8 & 4.5 & 4.7 & 99.3 & 100 & 64 & 90.6 & 4.2 & 7 \\
\hline & $Q_{S_{6}}$ & 5.8 & 5.3 & 5.3 & 5.7 & 43.2 & 71.8 & 4.8 & 4.4 & 5.2 & 6.3 & 7.2 & 10.5 \\
\hline & $Q_{S_{7}}$ & 5 & 4.9 & 6.2 & 3.2 & 44.7 & 72.5 & 4.9 & 5.2 & 5.6 & 3.4 & 8.8 & 17 \\
\hline & $Q_{D_{1}}$ & 4.2 & 5.6 & 82.2 & 99.3 & 73.9 & 99.1 & 6.6 & 13.9 & 18.4 & 38.5 & 5.2 & 8.1 \\
\hline & $Q_{D_{2}}$ & 4.2 & 5.3 & 87.3 & 99.8 & 70 & 98.3 & 21.8 & 50.5 & 25.9 & 59.5 & 4.7 & 7.8 \\
\hline & $Q_{D_{3}}$ & 5 & 4.9 & 6.4 & 5.3 & 6.3 & 5.3 & 99.5 & 100 & 67.5 & 94 & 100 & 100 \\
\hline & $Q_{D_{4}}$ & 5.8 & 4.9 & 83.9 & 99.4 & 76 & 98.5 & 11 & 18.6 & 21.3 & 41.6 & 9.4 & 17.6 \\
\hline & $Q_{O_{1}}$ & 4.1 & 5.5 & 77.1 & 98.7 & 64.7 & 97.4 & 97.6 & 100 & 62.7 & 91.4 & 100 & 100 \\
\hline & $Q_{O_{2}}$ & 5.6 & 4.7 & 80 & 99 & 9.4 & 15.2 & 99.5 & 100 & 71.8 & 96.9 & 100 & 100 \\
\hline & $Q_{O_{3}}$ & 5.5 & 4.4 & 73.1 & 97.9 & 62.6 & 96.1 & 99.4 & 100 & 67.5 & 94.5 & 100 & 100 \\
\hline & $Q_{O_{4}}$ & 4.5 & 4.9 & 85.5 & 99.8 & 65.2 & 97.3 & 18.7 & 44.5 & 22.7 & 50.7 & 5.7 & 8.4 \\
\hline \multirow[t]{15}{*}{0.8} & $Q_{S_{1}}$ & 5.8 & 4.5 & 3.2 & 5.5 & 61.4 & 100 & 53 & 92 & 2 & 2 & 0.9 & 1.2 \\
\hline & $Q_{S_{2}}$ & 3.9 & 4.6 & 52.3 & 84 & 46.9 & 83 & 3.8 & 2.7 & 10.9 & 23.5 & 7.9 & 19.7 \\
\hline & $Q_{S_{3}}$ & 4.1 & 4.4 & 76.3 & 97.1 & 55.5 & 88.1 & 27.5 & 58.9 & 28.5 & 59.2 & 34.3 & 72.8 \\
\hline & $Q_{S_{4}}$ & 5.2 & 4.5 & 4.4 & 6.3 & 3.9 & 5.1 & 100 & 100 & 88.6 & 100 & 99.6 & 100 \\
\hline & $Q_{S_{5}}$ & 5.7 & 5.3 & 4 & 5.9 & 3.4 & 4.5 & 100 & 100 & 85 & 100 & 43.1 & 66 \\
\hline & $Q_{S_{6}}$ & 6.3 & 5.8 & 4.7 & 5.2 & 76.5 & 96 & 65.1 & 87 & 6.4 & 9.1 & 18.7 & 24.3 \\
\hline & $Q_{S_{7}}$ & 5.4 & 4.6 & 4.7 & 5.1 & 74.9 & 96.2 & 60.9 & 86 & 6.7 & 7.9 & 7.3 & 5.3 \\
\hline & $Q_{D_{1}}$ & 4.8 & 5 & 40.5 & 72.8 & 90.1 & 100 & 31.5 & 80.7 & 6 & 15.3 & 4.6 & 8.3 \\
\hline & $Q_{D_{2}}$ & 5 & 5 & 67.7 & 97.7 & 95.9 & 100 & 50.2 & 95.4 & 20.7 & 49.4 & 19 & 47.7 \\
\hline & $Q_{D_{3}}$ & 4.1 & 5.2 & 2.9 & 4.1 & 4.6 & 5.5 & 100 & 100 & 82.6 & 100 & 100 & 100 \\
\hline & $Q_{D_{4}}$ & 4.7 & 5.6 & 59.2 & 88.8 & 100 & 100 & 83.2 & 99.5 & 27 & 52.3 & 33.9 & 61.4 \\
\hline & $Q_{O_{1}}$ & 5.7 & 4.8 & 33.4 & 69.4 & 86 & 99.9 & 100 & 100 & 76.5 & 98 & 95.8 & 99.9 \\
\hline & $Q_{O_{2}}$ & 4.6 & 5.3 & 37.6 & 70.8 & 31.1 & 68.1 & 100 & 100 & 85.4 & 100 & 99.8 & 100 \\
\hline & $Q_{O_{3}}$ & 5.5 & 6.1 & 30.5 & 60.6 & 83.8 & 99.8 & 100 & 100 & 78 & 98.2 & 99.6 & 100 \\
\hline & $Q_{O_{4}}$ & 9 & 5.9 & 71.6 & 97.6 & 99.5 & 100 & 61.8 & 94.6 & 24.4 & 56.3 & 59.9 & 92.4 \\
\hline
\end{tabular}

Note: The bold entries represent the sizes in percentage, the others are the empirical powers in percentage. The marginals are transformed by the empirical CDF's. The null hypothesis is that the data are generated according to a Gaussian copula. The nominal size in all cases is 5\%. The number of replications is 1, 000. 
where $\Phi^{-1}(\cdot)$ is the inverse of the standard normal distribution. The DGP is such that individual variables are conditionally normal and the copula is generated according to the Gaussian, $t$, Plackett, Gumbel, Clayton, Gumbel-Hougaard or asymmetric Gumbel-Hougaard copula.

We use a three-step estimator to construct the test statistic. First, we estimate the dynamic parameters of the model using the maximum likelihood estimator. We then estimate the marginal distributions of the standardized residuals via the empirical CDF's. In the third step, we estimate the coefficients of the null copula distribution using the maximum likelihood estimator. The test statistic is then constructed using the empirical CDF's and the estimated copula coefficients according to (20). Like the previous experiment, we set sample size $n=500,1000$ and repeat each experiment 1,000 times.

We report the simulation results in Table 3. The overall results are similar to those obtained for iid data in terms of size and power performance. As a matter of fact, the general patterns are essentially identical across these two tables. Therefore, our experiments confirm the remarkable finding of Chen and Fan (2006) that the asymptotic variance of the MLE of the copula parameters is not affected by the estimation of dynamic coefficients in the SCOMDY models. Thus our proposed copula specification tests can be applied to the dynamic models directly. Given that innovations in these models are properly standardized, the asymptotic results derived under the iid assumption are shown to be valid in these experiments.

\section{Empirical application}

In this section we present three illustrative examples to demonstrate the usefulness of our tests. We consider two cross-sectional datasets and one time series case. In each example, we first conduct the specification test. When the null hypothesis is rejected, we proceed to estimate an alternative copula function using diagnostic information provided by the test. Although in our simulations we only consider the Gaussian copula as the null distribution, here we provide an example where the more plausible null distribution is the Gumbel distribution, further demonstrating the flexibility of the proposed method.

\subsection{Uranium exploration data}

Our first example looks at the Uranium exploration data that have been investigated by Cook and Johnson $(1981,1986)$ and Genest et al. (2006). The dataset consists of 655 log-concentrations of seven chemical elements in water samples collected from the Montrose quadrangle of western Colorado (USA). Our interest is to understand the dependence in concentrations between the elements cesium (Cs) and scandium (Sc). Figure 1 displays the scatterplot of log concentrations of cesium and scandium. The graph suggests a strong positive left tail dependence between the concentration of $\mathrm{Cs}$ and Sc, but little right-tail dependence. There is also a clear diagonal asymmetry: More data 
Table 3: Empirical sizes and powers of the constructed tests (\%) in SCOMDY models

\begin{tabular}{|c|c|c|c|c|c|c|c|c|c|c|c|c|c|}
\hline \multirow[b]{2}{*}{$\rho$} & \multirow[b]{2}{*}{$C_{0}$} & \multicolumn{2}{|c|}{$C_{N}$} & \multicolumn{2}{|c|}{$C_{t_{4}}$} & \multicolumn{2}{|c|}{$C_{P}$} & \multicolumn{2}{|c|}{$C_{C}$} & \multicolumn{2}{|c|}{$C_{G}$} & \multicolumn{2}{|c|}{$C_{A G}$} \\
\hline & & $\mathrm{n}=500$ & 1000 & $\mathrm{n}=500$ & 1000 & $\mathrm{n}=500$ & 1000 & $\mathrm{n}=500$ & 1000 & $\mathrm{n}=500$ & 1000 & $\mathrm{n}=500$ & 1000 \\
\hline \multirow[t]{15}{*}{0.2} & $Q_{S_{1}}$ & 4.4 & 5.1 & 3.3 & 4.5 & 19.8 & 38.6 & 5.1 & 7.9 & 4.3 & 7 & 50.7 & 90.8 \\
\hline & $Q_{S_{2}}$ & 4 & 6.2 & 95.9 & 100 & 4.8 & 4.4 & 10.6 & 13.9 & 14.7 & 28.6 & 48.2 & 77.6 \\
\hline & $Q_{S_{3}}$ & 4.6 & 4.9 & 17.8 & 29.3 & 3.9 & 4.4 & 6.2 & 10.9 & 9.3 & 18.3 & 26.5 & 47.4 \\
\hline & $Q_{S_{4}}$ & 5 & 4.5 & 4.9 & 4.3 & 4.5 & 4.7 & 42.3 & 70.3 & 20 & 35.7 & 90.6 & 99.9 \\
\hline & $Q_{S_{5}}$ & 6.4 & 4.4 & 4.5 & 5.6 & 4 & 5.5 & 42.9 & 72.5 & 22.1 & 34.8 & 7.8 & 9.1 \\
\hline & $Q_{S_{6}}$ & 4.3 & 4.4 & 4.8 & 4.6 & 10.6 & 21.2 & 5.7 & 5.8 & 4.8 & 5.4 & 52.8 & 85.4 \\
\hline & $Q_{S_{7}}$ & 4.3 & 4.8 & 4.5 & 4.6 & 12.5 & 16.1 & 4.1 & 5.6 & 5.1 & 5.2 & 26.5 & 46.5 \\
\hline & $Q_{D_{1}}$ & 5.1 & 5.5 & 92.1 & 99.9 & 15 & 33.4 & 6.8 & 13 & 12 & 21.9 & 96.6 & 100 \\
\hline & $Q_{D_{2}}$ & 4.7 & 5.4 & 88.8 & 99.9 & 15.4 & 26.1 & 5.7 & 11.6 & 12.3 & 20.6 & 94.6 & 100 \\
\hline & $Q_{D_{3}}$ & 6.2 & 4.8 & 4.9 & 5.3 & 5.8 & 4.8 & 50.4 & 79.7 & 22.4 & 40.4 & 87.9 & 100 \\
\hline & $Q_{D_{4}}$ & 4.3 & 5 & 88.5 & 99.8 & 13.1 & 22.5 & 6.9 & 10.3 & 9.3 & 17.5 & 94.3 & 100 \\
\hline & $Q_{O_{1}}$ & 4.2 & 3.8 & 87.9 & 99.8 & 15 & 25.9 & 31 & 60 & 19.2 & 40 & 100 & 100 \\
\hline & $Q_{\mathrm{O}_{2}}$ & 4.3 & 4.4 & 87.9 & 99.8 & 4.7 & 6.2 & 49.2 & 81.9 & 26.8 & 49.5 & 95.2 & 100 \\
\hline & $Q_{O_{3}}$ & 4.8 & 5.4 & 85.4 & 99.4 & 14.2 & 24.3 & 40.9 & 78.4 & 23.3 & 47.1 & 100 & 100 \\
\hline & $Q_{O_{4}}$ & 5.1 & 5.2 & 84.8 & 99.8 & 10.9 & 23.3 & 8.4 & 10.5 & 8.2 & 18.8 & 92.9 & 100 \\
\hline \multirow[t]{15}{*}{0.5} & $Q_{S_{1}}$ & 4.3 & 4.4 & 2.8 & 3.2 & 65.5 & 91.8 & 0.6 & 1.5 & 4.4 & 5.1 & 5.9 & 14.1 \\
\hline & $Q_{S_{2}}$ & 5.2 & 5.7 & 91.2 & 99.5 & 13.9 & 25.1 & 13.5 & 22.9 & 26.1 & 53 & 3.2 & 3 \\
\hline & $Q_{S_{3}}$ & 5.3 & 4.8 & 62.2 & 95.1 & 6.9 & 8 & 36.1 & 65.8 & 30.6 & 57.6 & 4.6 & 4.8 \\
\hline & $Q_{S_{4}}$ & 4.9 & 5.2 & 5.2 & 4.2 & 4.5 & 4 & 99.8 & 100 & 60.9 & 89.4 & 100 & 100 \\
\hline & $Q_{S_{5}}$ & 4.3 & 5.8 & 4.6 & 5.4 & 4.9 & 6.2 & 99.4 & 100 & 62.2 & 90.1 & 5.4 & 5.3 \\
\hline & $Q_{S_{6}}$ & 5.2 & 4.6 & 5.3 & 5.2 & 43.7 & 73.5 & 5.4 & 6 & 4.1 & 4.5 & 5.4 & 12.7 \\
\hline & $Q_{S_{7}}$ & 4.5 & 6.4 & 4 & 5.2 & 41.3 & 71.5 & 4.9 & 4.8 & 6 & 4.9 & 8.6 & 17.6 \\
\hline & $Q_{D_{1}}$ & 4.2 & 3.9 & 82.2 & 99.6 & 75.7 & 98.8 & 7.3 & 13.1 & 16.8 & 41.3 & 3.4 & 7.6 \\
\hline & $Q_{D_{2}}$ & 4.1 & 5.5 & 86.7 & 99.7 & 69.3 & 98.6 & 21.8 & 47.2 & 25.7 & 55.8 & 6 & 6.5 \\
\hline & $Q_{D_{3}}$ & 5.7 & 4.9 & 4.6 & 4.4 & 4.4 & 5.5 & 99.5 & 100 & 62 & 91.5 & 100 & 100 \\
\hline & $Q_{D_{4}}$ & 6.3 & 5.4 & 86.6 & 99.6 & 74 & 97.1 & 11.2 & 19 & 20.6 & 41 & 8.7 & 13.7 \\
\hline & $Q_{O_{1}}$ & 6 & 5.4 & 77.7 & 98.6 & 68.7 & 97.6 & 97.6 & 99.9 & 59.1 & 89.8 & 100 & 100 \\
\hline & $Q_{\mathrm{O}_{2}}$ & 6.7 & 4.1 & 79.7 & 98.3 & 9 & 15.4 & 99.2 & 100 & 72 & 96.5 & 100 & 100 \\
\hline & $Q_{O_{3}}$ & 4.4 & 5 & 71.9 & 97.7 & 64.9 & 96.3 & 99 & 100 & 65 & 94 & 100 & 100 \\
\hline & $Q_{O_{4}}$ & 3.9 & 4.8 & 86.3 & 99.9 & 66.8 & 98.1 & 19.5 & 40 & 22.4 & 51.3 & 4.9 & 7.7 \\
\hline \multirow[t]{15}{*}{0.8} & $Q_{S_{1}}$ & 4.8 & 4.1 & 3.2 & 4.7 & 63.3 & 96.7 & 54.2 & 92.4 & 2.2 & 2.2 & 0.4 & 0.8 \\
\hline & $Q_{S_{2}}$ & 5.3 & 3.5 & 53.1 & 85.5 & 43.7 & 82.9 & 3.6 & 2.6 & 12.2 & 23.4 & 7.7 & 19.5 \\
\hline & $Q_{S_{3}}$ & 4.5 & 5 & 71.7 & 97.6 & 53.5 & 88.3 & 25.6 & 60.2 & 27.8 & 58.8 & 33.3 & 71.3 \\
\hline & $Q_{S_{4}}$ & 4.1 & 4.7 & 4.8 & 4.9 & 4 & 5 & 100 & 100 & 85.4 & 99 & 98.8 & 100 \\
\hline & $Q_{S_{5}}$ & 4.6 & 4.5 & 4.6 & 4 & 6.1 & 4.1 & 100 & 100 & 83.6 & 98.8 & 41.3 & 69 \\
\hline & $Q_{S_{6}}$ & 5.5 & 6.3 & 4.6 & 5.2 & 78.9 & 95 & 64.7 & 90 & 8.3 & 7.8 & 20.9 & 26 \\
\hline & $Q_{S_{7}}$ & 7 & 5.9 & 4.6 & 5.6 & 74.9 & 94.1 & 64.2 & 84.5 & 8.4 & 8.2 & 5.6 & 6.6 \\
\hline & $Q_{D_{1}}$ & 3.9 & 5.4 & 39.9 & 72.2 & 89.9 & 100 & 36.3 & 82.3 & 6.8 & 14.4 & 3.4 & 9.6 \\
\hline & $Q_{D_{2}}$ & 4 & 4.2 & 64.2 & 96.2 & 95.1 & 100 & 52.5 & 95.7 & 17.1 & 51.8 & 18.1 & 51.8 \\
\hline & $Q_{D_{3}}$ & 5.1 & 5.6 & 3.8 & 4.6 & 4.6 & 6 & 100 & 100 & 79.9 & 100 & 100 & 100 \\
\hline & $Q_{D_{4}}$ & 6 & 4.6 & 57.9 & 87.4 & 100 & 100 & 85.3 & 99.1 & 31.5 & 51.6 & 33.5 & 62.1 \\
\hline & $Q_{O_{1}}$ & 6.3 & 5.9 & 33.6 & 70.5 & 82.4 & 100 & 100 & 100 & 74.8 & 97.9 & 94.5 & 100 \\
\hline & $Q_{O_{2}}$ & 5.1 & 5.1 & 36.8 & 70.5 & 31.4 & 68.4 & 100 & 100 & 81.5 & 100 & 99.5 & 100 \\
\hline & $Q_{O_{3}}$ & 4.8 & 6.4 & 30.9 & 62.3 & 81.8 & 99.9 & 100 & 100 & 77.6 & 98.3 & 99.4 & 100 \\
\hline & $Q_{O_{4}}$ & 8.2 & 5.6 & 70.3 & 97.8 & 98 & 100 & 63.1 & 96.8 & 23.1 & 55.5 & 61.6 & 90.5 \\
\hline
\end{tabular}

Note: The bold entries represent the sizes in percentage, the others are the empirical powers in percentage. The marginals follow an $\operatorname{AR}(1)-G A R C H(1,1)$ DGP. The null hypothesis is that the copulas of the innovations belong to the Gaussian family. The nominal size in all cases is 5\%. The number of replications is $1,000$. 


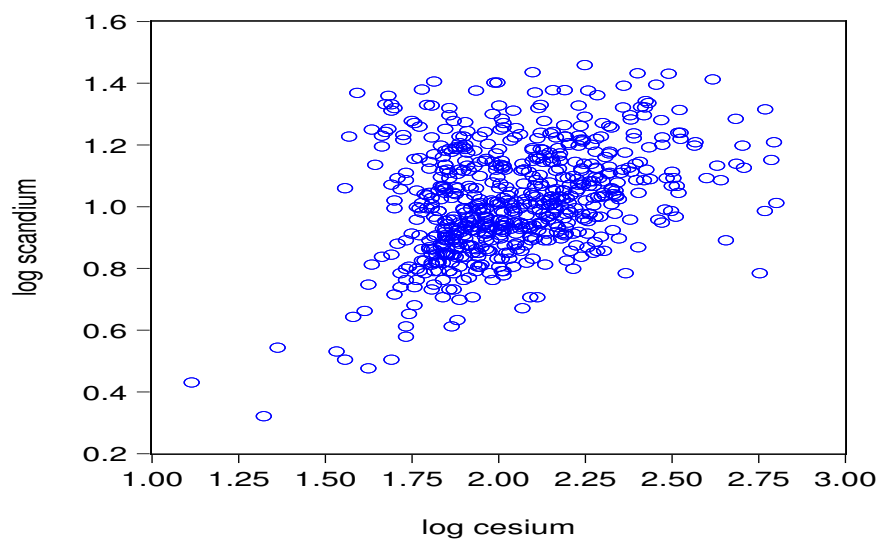

Figure 1: Scatterplot of log cesium and log scandium

points are clustered in the upper-left quadrant. Gaussian copula is expected to be rejected since it is radially symmetric and does not allow tail dependence.

We transformed the samples by their empirical distribution functions and tested the Gaussian copula null hypothesis on these variables. The two-step estimator of the correlation coefficient of the Gaussian copula, as defined in equation (11), is $\hat{\alpha}=0.3353$. The P-values of constructed tests are reported in Table 4. Not surprisingly, the null hypothesis of Gaussian copula is rejected at the $5 \%$ level by all tests except for a number of tests with a single moment function $\left(Q_{S_{1}}, Q_{S_{3}}, Q_{S_{6}}\right.$ and $\left.Q_{S_{7}}\right)$.

Table 4: Test results on the Uranium exploration data under the Gaussian null hypothesis

\begin{tabular}{lcccccccc}
\hline Test & $Q_{S_{1}}$ & $Q_{S_{2}}$ & $Q_{S_{3}}$ & $Q_{S_{4}}$ & $Q_{S_{5}}$ & $Q_{S_{6}}$ & $Q_{S_{7}}$ & $Q_{D_{1}}$ \\
\hline P-value & 0.8368 & $1.6435 \mathrm{e}-05$ & 0.0839 & $1.8042 \mathrm{e}-07$ & 0.0060 & 0.9851 & 0.0763 & $9.0238 \mathrm{e}-05$ \\
\hline Test & $Q_{D_{2}}$ & $Q_{D_{3}}$ & $Q_{D_{4}}$ & $Q_{O_{1}}$ & $Q_{O_{2}}$ & $Q_{O_{3}}$ & $Q_{O_{4}}$ & \\
\hline P-value & $1.8987 \mathrm{e}-04$ & $2.9196 \mathrm{e}-09$ & $2.1740 \mathrm{e}-05$ & $3.9380 \mathrm{e}-09$ & $1.0288 \mathrm{e}-10$ & $9.0367 \mathrm{e}-11$ & $5.4132 \mathrm{e}-04$ & \\
\hline
\end{tabular}

As is discussed above, the proposed tests are diagnostic for they provide useful guidance in the construction of alternative copula specifications. When the null hypothesis is rejected, we shall augment the null copula distribution with extra features suggested by the diagnostic tests. This alternative density is obtained by minimizing the Kullback-Leibler Information Criterion between the target density and the null density, subject to the side moment conditions associated with the set of features our test fails to reject. With the null distribution being the Gaussian copula $C_{N}$, the alternative density takes the form

$$
c_{g}\left(v_{1}, v_{2}\right)=c_{N}\left(v_{1}, v_{2} ; \hat{\alpha}\right) \exp \left(\hat{\lambda}^{T} \mathfrak{g}\left(v_{1}, v_{2} ; \hat{\alpha}\right)-\hat{\lambda}_{0}\right)
$$


with

$$
\hat{\lambda}_{0}=\log \int c_{N}\left(v_{1}, v_{2} ; \hat{\alpha}\right) \exp \left(\hat{\lambda}^{T} \mathfrak{g}\left(v_{1}, v_{2} ; \hat{\alpha}\right)\right) d v_{1} d v_{2}
$$

where $\mathfrak{g}$ is the set of moment functions involved in a given test.

For each test that fails to reject the null hypothesis, we can subsequently calculate an alternative copula density using the method of $\mathrm{Wu}$ (2010), incorporating the set of moment functions involved in the test. For instance, we denote by $c_{D_{1}}$ the alternative copula density corresponding with test $Q_{D_{1}}$. It follows that

$$
c_{D_{1}}\left(v_{1}, v_{2}\right)=c_{N}\left(v_{1}, v_{2} ; \hat{\alpha}\right) \exp \left(\hat{\lambda}_{1} \psi_{1}\left(v_{1}\right) \psi_{1}\left(v_{2}\right)+\hat{\lambda}_{2} \psi_{2}\left(v_{1}\right) \psi_{2}\left(v_{2}\right)-\hat{\lambda}_{0}\right)
$$

All other densities are constructed in a similar manner. We report the log likelihood and the AIC, BIC values of each estimated copula density in Table $5 .^{3}$ For comparison, we also report results on the null distribution. Although $c_{O_{3}}$ has a slightly higher log likelihood, both the AIC and BIC favor $c_{O_{2}}$. Note that $c_{O_{2}}$ contains three moment functions $\left(\psi_{1} \psi_{2}, \psi_{2} \psi_{1}, \psi_{2} \psi_{2}\right)$, while $c_{O_{3}}$ contains an additional term $\psi_{1} \psi_{1}$. Our results thus suggest that limited information content of $\psi_{1} \psi_{1}$, given that conveyed by $\left(\psi_{1} \psi_{2}, \psi_{2} \psi_{1}, \psi_{2} \psi_{2}\right)$, does not warrant its inclusion in the model. ${ }^{4}$

Figure 2 reports the contour plots of the Gaussian copula density $c_{N}$ with the correlation coefficient $\hat{\rho}=0.3353$ (left-hand panel) and the alternative copula density function $c_{\mathrm{O}_{2}}$ (right-hand panel). The Gaussian copula is radially symmetric. In contrast, the alternative copula density captures two salient features of the data: (i) The density at the lower-left corner is clearly higher than that at the upper-right corner, reflecting the asymmetric tail behaviors revealed in Figure 1; (ii) The density is apparently asymmetric about the diagonal, with a minor but non-negligible mode at the upper left corner, which is consistent with the unbalanced distribution of observations about the diagonal. It is reassuring to see that our test provides useful pointers for subsequent analysis when the null hypothesis is rejected. This is in contrast to omnibus tests, such as the Komogorov-Sminov test or Cramer-von-Mises test, which do not suggest possible directions of alternative specifications.

\footnotetext{
${ }^{3}$ The $A I C$ and $B I C$ are defined as, $A I C=-2 \log L+2 p, B I C=-2 \log L+p \log n$, where $\log L$ represents the $\log$-likelihood of the fitted model, $p$ is the number of parameters, and $n$ is the number of data points.

${ }^{4}$ Chen and Fan $(2005,2006)$ propose formal copula model selection procedures to test whether a model favored by an information criterion is significantly better than other candidates, wherein all competing models are allowed to be misspecified.
} 
Table 5: Estimation results of copula density functions for Uranium exploration data

\begin{tabular}{lccccccc}
\hline density & $c_{N}$ & $c_{S_{2}}$ & $c_{S_{4}}$ & $c_{S_{5}}$ & $c_{S_{7}}$ & $c_{D_{1}}$ & $c_{D_{2}}$ \\
\hline $\log L$ & 39.3483 & 46.9915 & 51.1782 & 43.0994 & 40.7911 & 48.2087 & 48.2519 \\
AIC & -76.6965 & -89.9830 & -98.3564 & -82.1987 & -77.5822 & -90.4175 & -88.5038 \\
BIC & -72.2119 & -81.0137 & -89.3871 & -73.2295 & -68.6130 & -76.9636 & -70.5653 \\
\hline density & $c_{D_{3}}$ & $c_{D_{4}}$ & $c_{O_{1}}$ & $c_{O_{2}}$ & $c_{O_{3}}$ & $c_{O_{4}}$ & \\
\hline $\log L$ & 62.079 & 53.5333 & 58.8750 & 70.2146 & 70.9438 & 58.8848 & \\
AIC & -118.158 & -99.0665 & -109.75 & -132.4292 & -131.8875 & -107.7696 & \\
BIC & -104.7041 & -81.1280 & -91.8114 & -114.4907 & -109.4644 & -85.3464 & \\
\hline
\end{tabular}
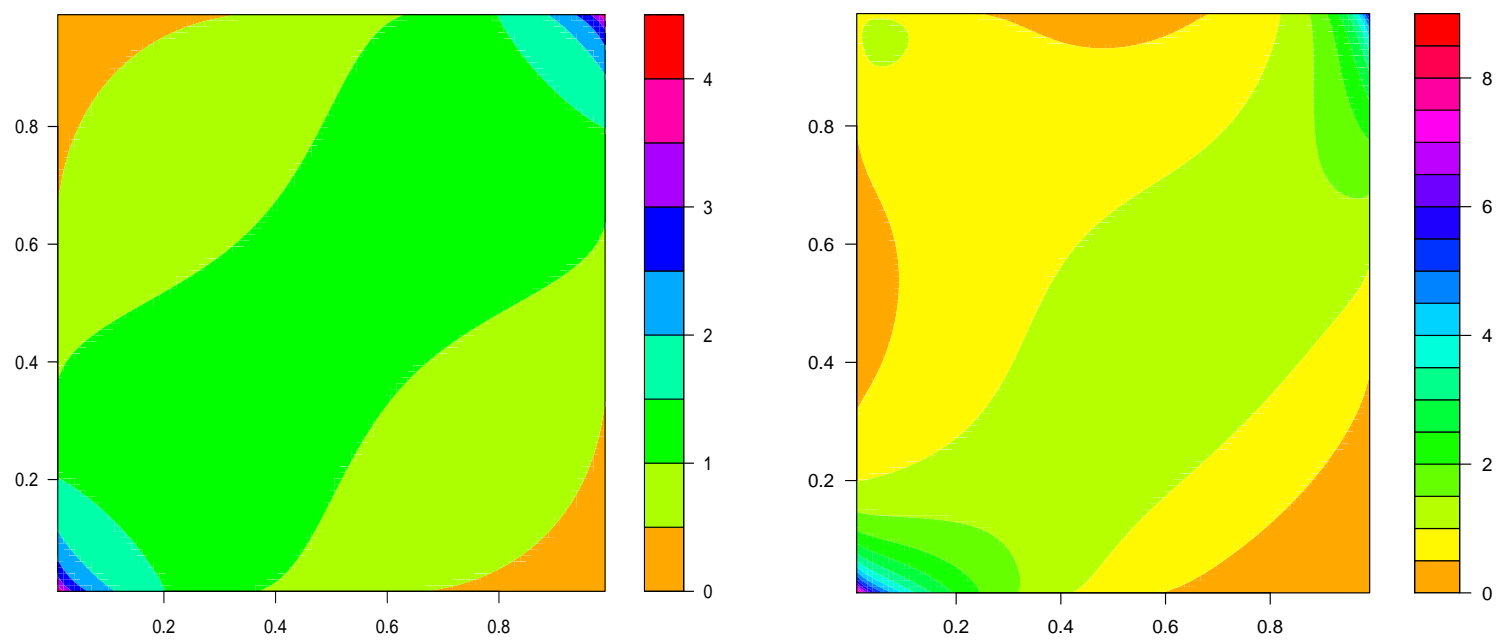

Figure 2: Contour plots of the Gaussian density function $c_{N}(\tilde{\alpha}=0.3353)$ (left panel) and of alternative copula density function $c_{\mathrm{O}_{2}}$ (right panel)

\subsection{Loss and ALAE data}

Our second example concerns the dependence structure of the indemnity payment(loss) and the allocated loss adjustment expense(ALAE) of insurance companies. The data were collected by the US Insurance Services Office and have been analyzed by Frees and Valdez (1998), Genest et al. (1995), Klugman and Parsa (1999), Denuit et al. (2004) and Chen and Fan (2005). A scatterplot of the loss and ALAE data on a log scale is depicted in Figure 3. There exsits apparent positive right-tail dependence between loss and ALAE: large losses tend to be associated with large ALAE's. On the other hand, there is no visible left-tail dependence between the two variables. As a result, 
Gumbel-Hougaard copula was chosen as the best fitting copula by Frees and Valdez (1998), Genest et al. (1998), Denuit et al. (2004) and Chen and Fan (2005). We refer readers to Frees and Valdez (1998) for a detailed description of this data set and to Chen and Fan (2005) for an overview of the copula model selection results of this data set in the literature. In keeping with the existing results, we use the Gumbel-Hougaard copula as the null hypothesis.

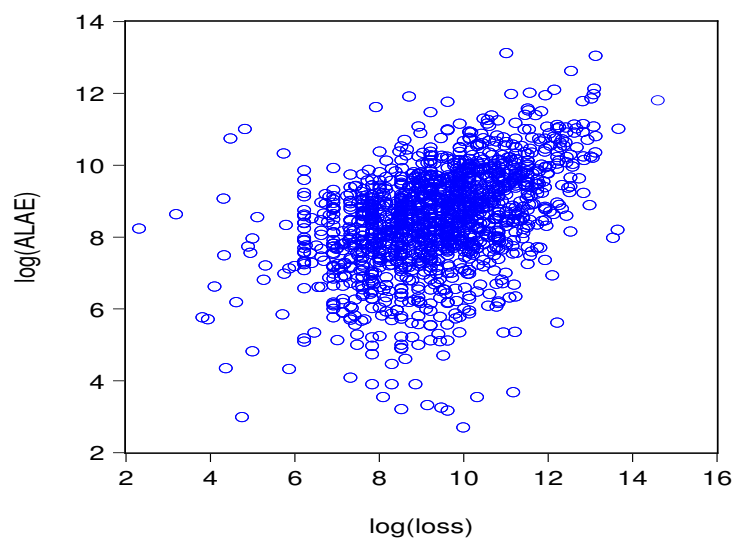

Figure 3: Scatterplot of loss and ALAE on a log scale

Following Chen and Fan (2005), we restrict ourselves to 1,466 uncensored data. We employ the same estimation and testing procedure as in the previous example on the current data set. The only difference is that now the baseline copula is the Gumbel-Hougaard copula. The testing results are reported in Table 6. It transpires that the previous studies have done a rather admirable job in selecting the Gumbel-Houggard copula: all tests, except $Q_{S_{3}}$, fail to reject the null hypothesis at the $5 \%$ confidence level. There are four other tests with $p$-values slightly less than $10 \%$. We proceed to construct alternative copula densities, considering all tests with $p$-values less than 0.1. The estimation results reported in Table 6 suggest that both the AIC and BIC rank the GumbelHougaard copula and $c_{S_{3}}$ as the two top choices; the Gumbel-Hougaard copula is preferred by the BIC, while $c_{S_{3}}$, which augments the Gumbel-Hougaard copula with one additional term, is preferred by the AIC. This discrepancy is attributed to the difference between the AIC and BIC: the BIC imposes a heavier penalty on model complexity and therefore favors simpler models.

Table 6: The P-values of constructed tests for the data on loss and ALAE under the Gumbel null hypothesis

\begin{tabular}{lcccccccc}
\hline Test & $Q_{S_{1}}$ & $Q_{S_{2}}$ & $Q_{S_{3}}$ & $Q_{S_{4}}$ & $Q_{S_{5}}$ & $Q_{S_{6}}$ & $Q_{S_{7}}$ & $Q_{D_{1}}$ \\
\hline P-value & 0.1042 & 0.1973 & 0.0412 & 0.2229 & 0.2579 & 1.0000 & 1.0000 & 0.2287 \\
\hline Test & $Q_{D_{2}}$ & $Q_{D_{3}}$ & $Q_{D_{4}}$ & $Q_{O_{1}}$ & $Q_{O_{2}}$ & $Q_{O_{3}}$ & $Q_{O_{4}}$ & \\
\hline P-value & 0.1543 & 0.0956 & 0.6281 & 0.0849 & 0.0924 & 0.0769 & 0.2459 & \\
\hline
\end{tabular}


Table 7: Estimation results of the copula density functions for loss and ALAE data

\begin{tabular}{lcccccc}
\hline density & $c_{G}$ & $c_{S_{3}}$ & $c_{D_{3}}$ & $c_{O_{1}}$ & $c_{O_{2}}$ & $c_{O_{3}}$ \\
\hline $\log L$ & 190.7412 & 192.2133 & 192.0615 & 192.6459 & 193.0386 & 193.3354 \\
AIC & -379.4824 & -380.4265 & -378.1231 & -377.2918 & -378.0773 & -378.6708 \\
BIC & -374.1921 & -369.8459 & -362.2522 & -356.1307 & -356.9161 & -357.5096 \\
\hline
\end{tabular}

Figure 4 plots the estimated Gumbel copula density (with $\hat{\alpha}=1.4394$ ) and $c_{S_{3}}$ copula density. Both densities exhibit strong right tail dependence and relatively weak left tail dependence. There exists some subtle difference between the two densities in that the densities at the two extreme corners of $c_{S_{3}}$ density are slightly lower than those of the Gumbel copula. In summary, our tests indicate that the Gumble copula, as is selected by most existing studies, provides a satisfactory fit to the loss and ALAE data. Although the AIC favors an alternative copula specification, our exploratory analysis suggests the difference between these two is rather minor. This example on Gumbel copula demonstrates the flexibility of the proposed test and that it can be used for both exploratory and confirmatory purposes in data analysis.
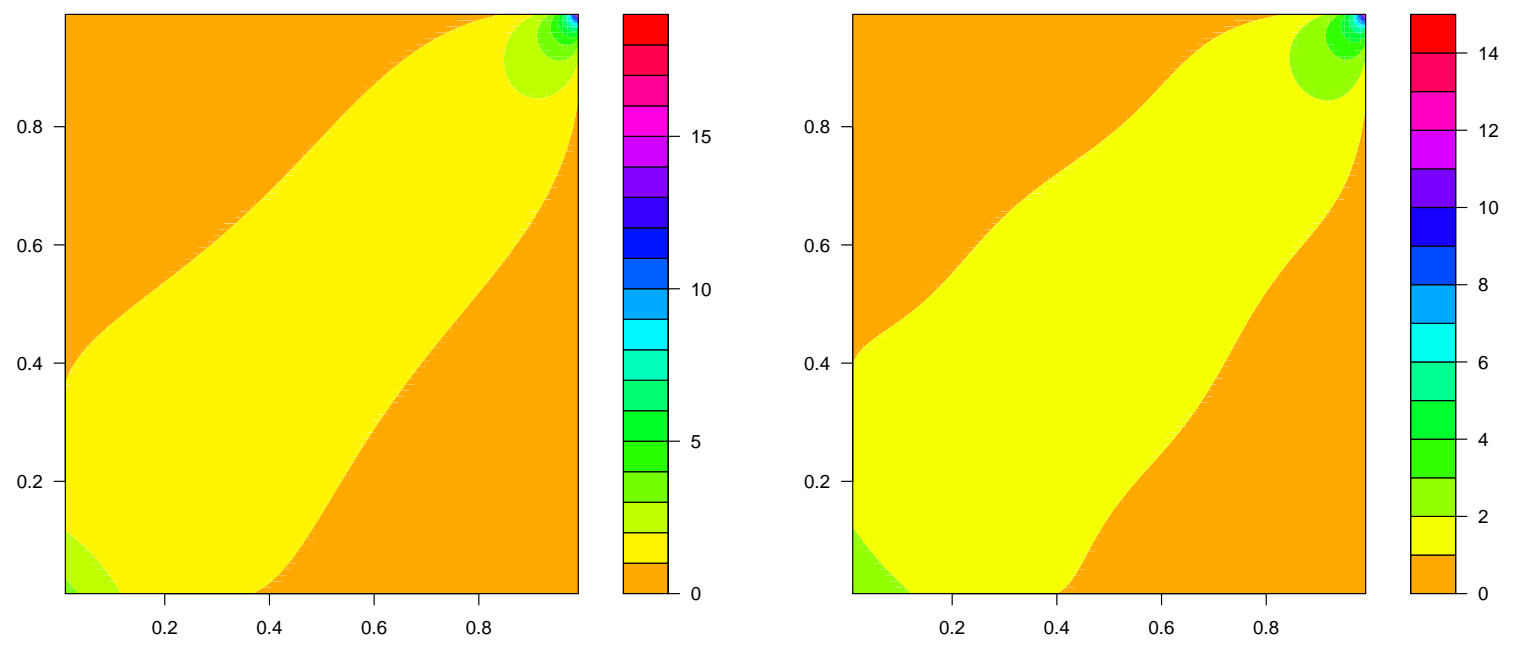

Figure 4: Contour plots of the Gumbel density function $c_{G}(\hat{\alpha}=1.4394)$ (left panel) and of alternative copula density function $c_{S_{3}}$ (right panel)

\subsection{Stock return example}

Lastly we apply our test to time series data, examining the dynamic relationship between the monthly SP500 Index and Hengseng Index of Hong Kong. Our sample covers the period from 


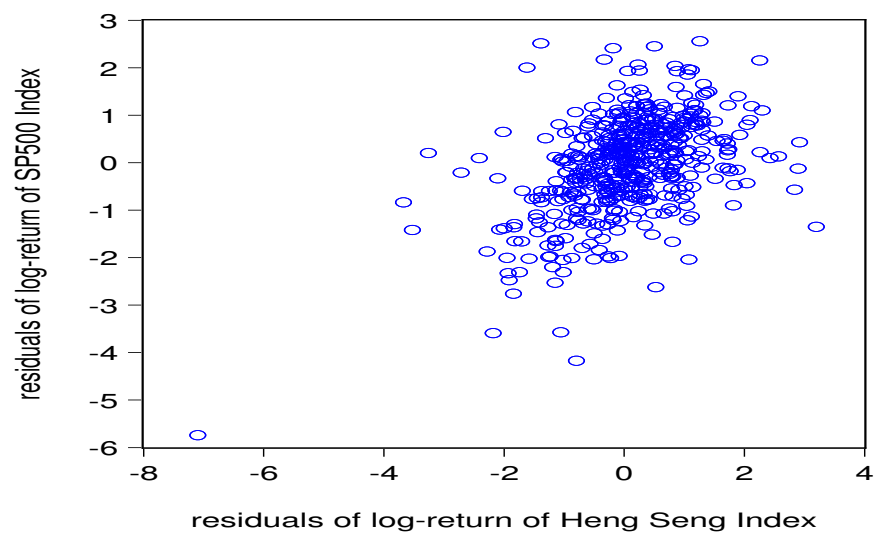

Figure 5: Scatterplot of the residuals of log-turns of SP500 Index and Hengseng Index

January 1970 till October, 2012 with 513 observations.

For each index, the stock return at time $t$ is calculated as $R_{t}=\log P_{t}-\log P_{t-1}$. We assume a GARCH $(1,1)$ model such that $R_{t}=\mu+e_{t}$, where $e_{t} \sim N\left(0, h_{t}\right)$, and $h_{t}=\gamma+\alpha e_{t-1}^{2}+\beta h_{t-1}$. The standardized residuals from the $\operatorname{GARCH}(1,1)$ models are used in subsequent estimation and testing. Figure 5 displays the scatterplot of the standardized residuals of two series. One can see that the U.S. and the HK markets exhibit considerable left tail dependence but smaller right tail dependence.

We transformed the standardized residuals by their empirical distribution functions and tested null hypothesis of the Gaussian copula distribution. The three-step estimator of the Gaussian yields $\hat{\alpha}=0.4496$. The $P$-values of the tests are reported in Table 8 . Six tests reject the null hypothesis of Gaussian copula at the $5 \%$ level. All tests rejecting the null hypothesis contain off-diagonal entries in the form of $\psi_{i} \psi_{j}, i \neq j, i, j=1,2$, which can be explained by the evident asymmetry in the two extreme tails revealed in Figure 5.

Table 8: Tests on log returns of SP500 Index and Hengseng Index under the Gaussian null hypothesis

\begin{tabular}{lcccccccc}
\hline Test & $Q_{S_{1}}$ & $Q_{S_{2}}$ & $Q_{S_{3}}$ & $Q_{S_{4}}$ & $Q_{S_{5}}$ & $Q_{S_{6}}$ & $Q_{S_{7}}$ & $Q_{D_{1}}$ \\
\hline P-value & 0.2070 & 0.0751 & 0.1222 & $1.1522 \mathrm{e}-04$ & 0.0139 & 0.2123 & 0.7737 & 0.0660 \\
\hline Test & $Q_{D_{2}}$ & $Q_{D_{3}}$ & $Q_{D_{4}}$ & $Q_{O_{1}}$ & $Q_{O_{2}}$ & $Q_{O_{3}}$ & $Q_{O_{4}}$ & \\
\hline P-value & 0.0620 & $2.4396 \mathrm{e}-04$ & 0.0993 & $1.4194 \mathrm{e}-05$ & $6.2826 \mathrm{e}-05$ & $2.1434 \mathrm{e}-05$ & 0.0777 & \\
\hline
\end{tabular}

We next construct alternative copula density functions associated with those tests that reject the null hypothesis at the $10 \%$ level. The estimation results are reported in Table 9. Although $c_{D_{3}}, c_{O_{1}}, c_{O_{2}}$ and $c_{O_{3}}$ have slightly higher log likelihood, both the AIC and BIC suggest $c_{S_{4}}$ as the 
preferred specification. Note that $c_{S_{4}}$ only contains a single moment function $\left(\psi_{1} \psi_{2}\right)$ while $c_{D_{3}}$, $c_{O_{1}}, c_{O_{2}}$ and $c_{O_{3}}$ contain one or more additional terms. Our results suggest that these extra terms do not contain sufficiently useful information given the term $\psi_{1} \psi_{2}$ already incorporated in $c_{S_{4}}$.

The contours of the estimated Gaussian copula $(\hat{\alpha}=0.4496)$ and $c_{S_{4}}$ are reported in Figure 6 . Two differences are revealed by the plots. First, the Gaussian copula fails to capture the radial asymmetry in the data. In contrast, the alternative copula density demonstrates a considerably higher density at the lower tail. Second, the alternative copula density exhibits a narrower and slightly asymmetric 'ridge' along the diagonal. Apparently these richer features are missed by the Gaussian copula, which is restrictive for it is parameterized by a single parameter. Our confidence in these implied features is supported by the strong statistical evidence conveyed in the reported specification tests and goodness-of-fit measures.

Table 9: Estimation results of the copula density function for stock return data

\begin{tabular}{lcccccc}
\hline density & $c_{N}$ & $c_{S_{2}}$ & $c_{S_{4}}$ & $c_{S_{5}}$ & $c_{D_{1}}$ & $c_{D_{2}}$ \\
\hline $\log L$ & 57.9164 & 59.0539 & 63.0573 & 60.0416 & 59.0713 & 59.3628 \\
AIC & -113.8327 & -114.1077 & -122.1146 & -116.0833 & -112.1426 & -110.7256 \\
BIC & -109.5924 & -105.6272 & -113.6341 & -107.6027 & -99.4218 & -93.7645 \\
\hline density & $c_{D_{3}}$ & $c_{D_{4}}$ & $c_{O_{1}}$ & $c_{O_{2}}$ & $c_{O_{3}}$ & $c_{O_{4}}$ \\
\hline $\log L$ & 63.3103 & 61.4497 & 63.9285 & 64.0315 & 64.0916 & 61.3160 \\
AIC & -120.6206 & -114.8994 & -119.8569 & -120.063 & -118.1832 & -112.6319 \\
BIC & -107.8998 & -97.9383 & -102.8958 & -103.1019 & -96.9818 & -91.4305 \\
\hline
\end{tabular}

\section{Concluding Remarks}

We have proposed moment-based tests of copula specifications for semiparametric copula models. Our tests can be characterized as score tests on moment conditions of empirical copula distributions under the null hypothesis. The tests are both flexible and easy to implement for it does not require selection of smoothing parameters. It is distribution free and therefore no simulations are needed for the critical values. In addition, our tests are diagnostic: when a set of moment conditions are not rejected by our specification test, they provide useful pointers for the construction of alternative copula densities. Our Monte Carlo simulations and empirical examples demonstrate the efficacy and usefulness of our method.

We conclude this study with some possible directions for future study. First, following Chen and Fan (2006) our extension to time series model maintains that the innovations of the SCOMDY models are iid. Further generalizations to accommodate inter temporally correlated innovations or time varying copulas may be of interest. Second, a common practice in the applications of Neyman's smooth test is to restrict the number of moments to a small number (usually no greater than 4). 

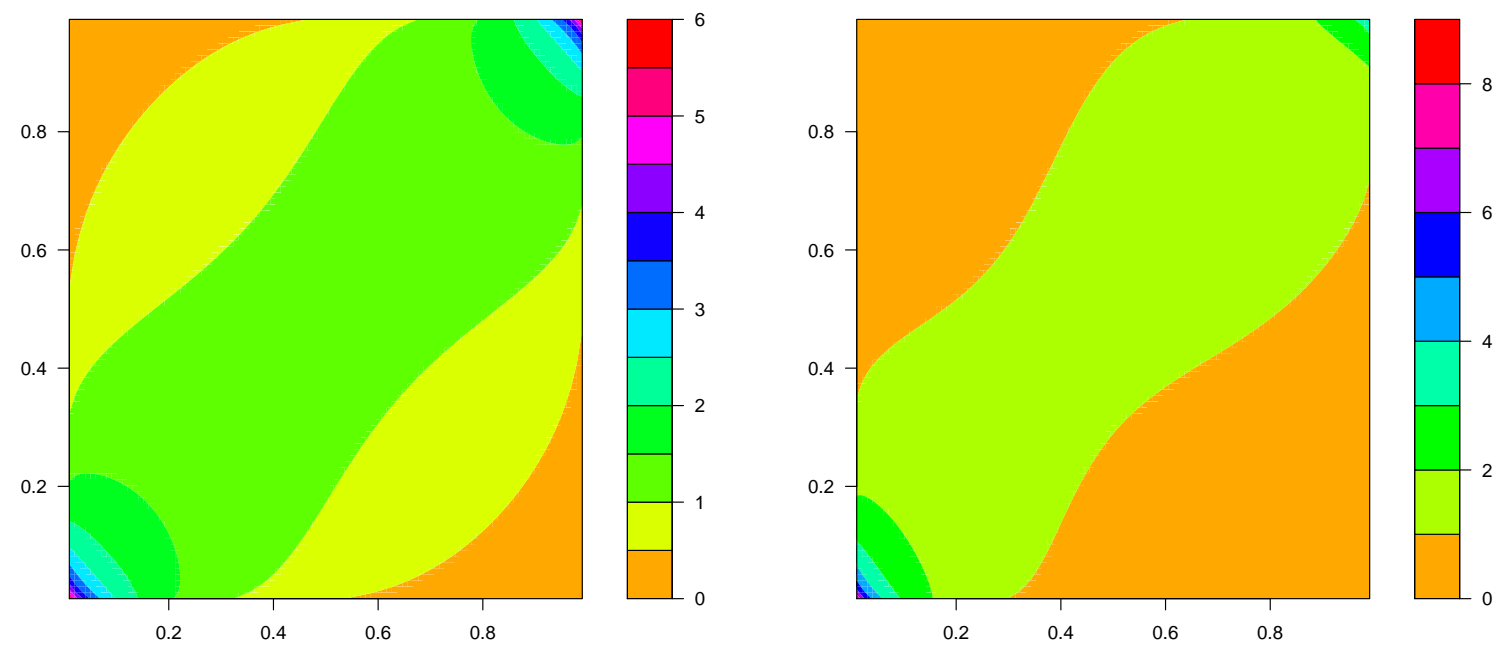

Figure 6: Contour plots of the Gaussian density function $c_{N}(\hat{\alpha}=0.4496)$ (left panel) and of alternative copula density function $c_{S_{4}}$ (right panel)

Ledwina (1994) and Kallenberg and Ledwinan (1995) study a modified Neyman's test wherein the number of moments is data-driven. Generalization of our tests along this line of thought is expected to lead to further improvements. Lastly, Chen et al. (2010) proposes the pseudo-likelihood ratio tests for semiparametric multivariate copula model selection subject to general censorship. Our tests can also be generalized in the same direction.

\section{References}

Bera, A. K., A. Ghosh, and Z. Xiao (2012). A smooth test for the equality of distributions. Econometric Theory FirstView, 1-28.

Berg, D. (2009). Copula goodness-of-fit testing: an overview and power comparison. European Journal of Finance 15, 675-701.

Chan, N., J. Chen, X. Chen, Y. Fan, and L. Peng (2009). Statistical inference for multivariate residual copula of garch models. Statistica Sinica 19(1), 53.

Chen, X. and Y. Fan (2005). Pseudo-likelihood ratio tests for semiparametric multivariate copula model selection. Canadian Journal of Statistics 33(3), 389-414.

Chen, X. and Y. Fan (2006). Estimation and model selection of semiparametric copula-based 
multivariate dynamic models under copula misspecification. Journal of Econometrics 135(1), $125-154$.

Chen, X., Y. Fan, and A. Patton (2004). Simple tests for models of dependence between multiple financial time series, with applications to us equity returns and exchange rates. London Economics Financial Markets Group Working Paper (483).

Chen, X., Y. Fan, D. Pouzo, and Z. Ying (2010). Estimation and model selection of semiparametric multivariate survival functions under general censorship. Journal of Econometrics 157(1), 129142.

Chen, Y. (2007). Moment-based copula tests for financial returns. Journal of Business 85 Economic Statistics 25(4), 377-397.

Cherubini, U., E. Luciano, and W. Vecchiato (2004). Copula methods in finance. Wiley.

Cherubini, U., S. Mulinacci, F. Gobbi, and S. Romagnoli (2011). Dynamic Copula Methods in Finance, Volume 625. Wiley.

Cook, R. and M. Johnson (1981). A family of distributions for modelling non-elliptically symmetric multivariate data. Journal of the Royal Statistical Society. Series B (Methodological), 210-218.

Cook, R. and M. Johnson (1986). Generalized burr-pareto-logistic distributions with applications to a uranium exploration data set. Technometrics 28(2), 123-131.

Denuit, M., O. Purcaru, and I. Van Keilegom (2004). Bivariate archimedean copula modelling for loss-alae data in non-life insurance. IS Discussion Papers 423.

Efron, B. and R. Tibshirani (1996). Using specially designed exponential families for density estimation. The Annals of Statistics 24(6), 2431-2461.

Fermanian, J. (2005). Goodness-of-fit tests for copulas. Journal of Multivariate Analysis 95, 119152.

Fermanian, J. (2012). An overview of the goodness-of-fit test problem for copulas. Working Paper, CREST.

Frees, E. and E. Valdez (1998). Understanding relationships using copulas. North American actuarial journal 2(1), 1-25.

Genest, C., K. Ghoudi, and L. Rivest (1995). A semiparametric estimation procedure of dependence parameters in multivariate families of distributions. Biometrika 82(3), 543-552. 
Genest, C., K. Ghoudi, and L. Rivest (1998). Comment on the paper by E. W. Frees and E. A. Valdez entitled "Understanding relationships using copulas.". North American Actuarial Journal 2, 143-149.

Genest, C., J. QUESSY, and B. Rémillard (2006). Goodness-of-fit procedures for copula models based on the probability integral transformation. Scandinavian Journal of Statistics 33(2), 337366.

Genest, C., B. Rémillard, and D. Beaudoin (2009). Goodness-of-fit tests for copulas: A review and a power study. Insurance: Mathematics and Economics 44(2), 199-213.

Janssen, A. (2000). Global power functions of goodness of fit tests. Annals of Statistics, 239-253.

Kallenberg, W. and T. Ledwinan (1995). Consistency and monte carlo simulation of data driven version of smooth goodness of fit tests. Annals of Statistics 23, 1594-1608.

Khoudraji, A. (1995). Contributions à l'étude des copules et à la modélisation des valeurs extrêmes bivariées. Ph.D. thesis, Université Laval, Québec, Canada.

Klugman, S. and R. Parsa (1999). Fitting bivariate loss distributions with copulas. Insurance: Mathematics and Economics 24(1), 139-148.

Ledwina, T. (1994). Data driven version of the neyman smooth test of fit. Journal of American Statistical Association 89, 1000-1005.

Li, D. and L. Peng (2009). Goodness-of-fit test for tail copulas modeled by elliptical copulas. Statistics 83 Probability Letters 79(8), 1097-1104.

Manner, H. and O. Reznikova (2012). A survey of time-varying copulas: specification, simulation and app. Econometric Reviews 31, 654-687.

McNeil, A., R. Frey, and P. Embrechts (2005). Quantitative risk management: concepts, techniques, and tools. Princeton university press.

Nelsen, R. B. (1993). Some concepts of bivariate symmetry. Journal of Nonparametric Statistics 3(1), 95-101.

Neyman, J. (1937). 'smooth test' for goodness of fit. Skandinaviske Aktuarietidskrift 20, 150-199.

Patton, A. (2012). A review of copula models for economic time series. Journal of Multivariate Analysis 110, 4-18.

Prokhorov, A. and P. Schmidt (2009). Likelihood-based estimation in a panel setting: Robustness, redundancy and validity of copulas. Journal of Econometrics 153, 93-104. 
Rayner, J. and D. Best (1990). Smooth tests of goodness of fit: an overview. International Statistical Review 58, 9-17.

Rémillard, B. (2010). Goodness-of-fit tests for copulas of multivariate time series. Working Paper, HEC Montral, Canada.

Sklar, A. (1959). Fonctions de rpartition n dimensions et leurs marges. Publications de l'Institut de Statistique de l'Universit de Paris 8, 229-231.

Thomas, D. R. and D. A. Pierce (1979). Neyman's smooth goodness-of-fit test when the hypothesis is composite. Journal of the American Statistical Association 74(366), pp. 441-445.

Wu, X. (2010). Exponential series estimator of multivariate densities. Journal of Econometrics $156(2), 354-366$.

\section{Appendix}

We first list some conditions needed to establish the large sample properties of the proposed tests.

Condition 1. $\mathrm{E}\left[l\left(U_{1 t}, U_{2 t} ; \alpha\right)\right]$ has a unique maximum $\alpha_{0} \in \operatorname{int}(\mathcal{A})$, where $\mathcal{A}$ is a compact subset of $\mathbb{R}^{p}$.

Condition 2. The true (unknown) copula function $c\left(v_{1}, v_{2}\right)$ has continuous partial derivatives with respect to $v_{1}$ and $v_{2}$.

Condition 3. For any $\left(v_{1}, v_{2}\right) \in(0,1)^{2}, l\left(v_{1}, v_{2} ; \alpha\right)$ is a continuous function of $\alpha$; $\left[\sup _{\alpha \in \mathcal{A}}\left|l\left(U_{1 t}, U_{2 t} ; \alpha\right)\right|\right]<$ $\infty$.

Condition 4. $B \equiv-\mathrm{E}\left[l_{\alpha \alpha}\left(U_{1 t}, U_{2 t} ; \alpha_{0}\right)\right]$ is positive definite; $\Sigma \equiv \operatorname{var}\left[l_{\alpha}\left(U_{1 t}, U_{2 t} ; \alpha_{0}\right)+\sum_{j=1}^{2} W_{j}\left(U_{j t} ; \alpha_{0}\right)\right]$ is finite and positive definite.

Condition 5. For $j=1,2, l_{\alpha j}\left(v_{1}, v_{2} ; \alpha_{0}\right)$ is well-defined and continuous in $\left(v_{1}, v_{2}\right) \in(0,1)^{2}$.

Condition 6. (i) $\left\|l_{\alpha}\left(v_{1}, v_{2} ; \alpha_{0}\right)\right\| \leq$ constant $\times \prod_{j=1}^{2}\left\{v_{j}\left(1-v_{j}\right)\right\}^{-a_{j}}$ for some $a_{j} \geq 0$ such that

$$
\mathrm{E}\left[\prod_{j=1}^{2}\left\{U_{j t}\left(1-U_{j t}\right)\right\}^{-2 a_{j}}\right]<\infty
$$

(ii) $\left\|l_{\alpha j}\left(v_{1}, v_{2} ; \alpha_{0}\right)\right\| \leq$ constant $\times\left\{v_{j}\left(1-v_{j}\right)\right\}^{-b_{j}}\left\{v_{3-j}\left(1-v_{3-j}\right)\right\}^{-a_{3-j}}$ for some $b_{j}>a_{j}$ such that

$$
\mathrm{E}\left[\left\{U_{j t}\left(1-U_{j t}\right)\right\}^{\xi_{t}-b_{t}}\left\{U_{3-j, t}\left(1-U_{3-j, t}\right)^{-a_{3-j}}\right\}\right]<\infty
$$

for some $\xi_{t} \in(0,1 / 2)$. 
Condition 7. For any $\left(v_{1}, v_{2}\right) \in(0,1)^{2}, l_{\alpha \alpha}\left(v_{1}, v_{2} ; \alpha\right)$ is a continuous function of $\alpha$ in a neighborhood of $\alpha_{0} ; \mathrm{E}\left[\sup _{\alpha \in \mathcal{A}:\left\|\alpha-\alpha_{0}\right\|=o(1)}\left\|l_{\alpha \alpha}\left(U_{1 t}, U_{2 t} ; \alpha\right)\right\|\right]<\infty$.

The consistence and asymptotic normality of $\hat{\alpha}$ is established by Genest et al. (1995) and Chen and Fan (2005). A sketch of the proof is given below.

Proof of Theorem 1. Under Conditions 1-3, the consistence of $\hat{\alpha}$ is readily obtained according to Proposition 1 of Chen and Fan (2005). Next, under Conditions 1-7, the asymptotic normality of $\hat{\alpha}$ is given by Proposition 2 of Chen and Fan (2005). Condition 6 allows the score function and its partial derivatives with respect to $v_{j}, j=1,2$ to blow up at the boundaries. The condition characterizes many popular copula functions such as the Gaussian, $t$ and Clayton copulas.

Condition 8. For any $\left(v_{1}, v_{2}\right) \in(0,1)^{2}, g\left(v_{1}, v_{2}\right)$ has continuous partial derivatives with respect to $v_{1}$ and $v_{2} ; \mathfrak{g}\left(v_{1}, v_{2} ; \alpha\right)$ is a continuous function of $\alpha ; \mathrm{E}\left[\sup _{\alpha \in \mathcal{A}}\left|\mathfrak{g}\left(U_{1 t}, U_{2 t} ; \alpha\right)\right|\right]<\infty$.

Condition 9. $\Omega=\operatorname{var}\left[\mathfrak{g}\left(U_{1 t}, U_{2 t} ; \alpha_{0}\right)+\sum_{j=1}^{2} Z_{j}\left(U_{j t}\right)+G^{T} B^{-1}\left\{l_{\alpha}\left(U_{1 t}, U_{2 t} ; \alpha_{0}\right)+\sum_{j=1}^{2} W_{j}\left(U_{j t} ; \alpha_{0}\right)\right\}\right]$ is finite and positive definite.

Condition 10. For any $\left(v_{1}, v_{2}\right) \in(0,1)^{2}, \frac{\partial}{\partial \alpha} \mu(g ; \alpha)$ is a continuous function of $\alpha$ in a neighborhood of $\alpha_{0} ; \sup _{\alpha \in \mathcal{A}:\left\|\alpha-\alpha_{0}\right\|=o(1)}\left\|\frac{\partial}{\partial \alpha} \mu(g ; \alpha)\right\|<\infty$.

Proof of Theorem 2. By Theorem 1, we have $\left\|\hat{\alpha}-\alpha_{0}\right\|=o_{p}(1)$. Applying Taylor's expansion to $\hat{\mathfrak{g}}(\hat{\alpha})$ with respect to $\hat{\alpha}$ at $\alpha_{0}$ yields

$$
\hat{\mathfrak{g}}(\hat{\alpha})=\frac{1}{n} \sum_{t=1}^{n} \mathfrak{g}\left(\tilde{U}_{1 t}, \tilde{U}_{2 t} ; \alpha_{0}\right)+\frac{1}{n} \sum_{t=1}^{n} \frac{\partial}{\partial \alpha} \mu(g ; \bar{\alpha})\left(\hat{\alpha}-\alpha_{0}\right)
$$

where $\bar{\alpha}$ is between $\alpha_{0}$ and $\hat{\alpha}$. Under Condition 10, we have

$$
\sup _{\alpha \in \mathcal{A}:\left\|\alpha-\alpha_{0}\right\|=o(1)}\left\|\frac{\partial}{\partial \alpha} \mu(g ; \alpha)-G\right\|=o(1) .
$$

It follows that (A.1) can be written as

$$
\hat{\mathfrak{g}}(\hat{\alpha})=\frac{1}{n} \sum_{t=1}^{n} \mathfrak{g}\left(\tilde{U}_{1 t}, \tilde{U}_{2 t} ; \alpha_{0}\right)+G^{T}\left(\hat{\alpha}-\alpha_{0}\right)+o_{p}(1) .
$$

Next Theorem 1 indicates that $\hat{\alpha}$ can be expressed as an asymptotically linear estimator such that

$$
\hat{\alpha}-\alpha_{0}=B^{-1} \frac{1}{n} \sum_{t=1}^{n} l_{\alpha}\left(\tilde{U}_{1 t}, \tilde{U}_{2 t} ; \alpha_{0}\right)+o_{p}(1) .
$$


Plugging A.3 into A.2 yields

$$
\hat{\mathfrak{g}}(\hat{\alpha})=\frac{1}{n} \sum_{t=1}^{n}\left[\mathfrak{g}\left(\tilde{U}_{1 t}, \tilde{U}_{2 t} ; \alpha_{0}\right)+G^{T} B^{-1} l_{\alpha}\left(\tilde{U}_{1 t}, \tilde{U}_{2 t} ; \alpha_{0}\right)\right]+o_{p}(1) \equiv \hat{R}+o_{p}(1) .
$$

Define

$$
J\left(v_{1}, v_{2}\right)=\mathfrak{g}\left(v_{1}, v_{2} ; \alpha_{0}\right)+G^{T} B^{-1} l_{\alpha}\left(v_{1}, v_{2} ; \alpha_{0}\right) .
$$

We then have

$$
\hat{R}=\frac{1}{n} \sum_{t=1}^{n} J\left(\tilde{U}_{1 t}, \tilde{U}_{2 t}\right)=\int J\left(v_{1}, v_{2}\right) d \tilde{C}\left(v_{1}, v_{2}\right),
$$

where $\tilde{C}$ is the rescaled empirical copula function given by

$$
\tilde{C}\left(v_{1}, v_{2}\right)=\frac{1}{n} \sum_{t=1}^{n} 1\left(\tilde{U}_{1 t} \leq v_{1}\right) 1\left(\tilde{U}_{2 t} \leq v_{2}\right)
$$

Using Proposition A.1(i) of Genest et al. (1995) or Lemma 1 of Chen and Fan (2005), we then have under Conditions 1-3 and 8, $\hat{R} \stackrel{p}{\rightarrow} \mathrm{E}[\hat{R}] \equiv \mathrm{E}\left[\mathfrak{g}\left(v_{1}, v_{2} ; \alpha_{0}\right)+G^{T} B^{-1} l_{\alpha}\left(v_{1}, v_{2} ; \alpha_{0}\right)\right]=0$. It follows that $\hat{\mathfrak{g}}(\hat{\alpha}) \stackrel{p}{\rightarrow} \mathrm{E}\left[\mathfrak{g}\left(\alpha_{0}\right)\right]=0$ as $n \rightarrow \infty$.

Next using Proposition A.1(ii) of Genest et al. (1995) or Lemma 2 of Chen and Fan (2005), we then have under Conditions 1-10,

$$
\sqrt{n} \int J\left(v_{1}, v_{2}\right) d\left\{\tilde{C}\left(v_{1}, v_{2}\right)-C\left(v_{1}, v_{2}\right)\right\} \stackrel{d}{\rightarrow} \mathcal{N}\left(0, \sigma^{2}\right)
$$

with

$$
\sigma^{2} \equiv \operatorname{var}\left[J\left(U_{1 t}, U_{2 t}\right)+\sum_{j=1}^{2} \int \frac{\partial}{\partial v_{j}} J\left(v_{1}, v_{2}\right) 1\left(U_{j t} \leq v_{j}\right) d C\left(v_{1}, v_{2}\right)\right] .
$$

$\Omega$ is then obtained by applying the variance formula (A.5) to (A.7), using also the following result:

$$
\int \frac{\partial}{\partial v_{j}} J\left(v_{1}, v_{2}\right) 1\left(U_{j t} \leq v_{j}\right) d C_{0}\left(v_{1}, v_{2} ; \alpha_{0}\right)=G^{T} B^{-1} \mathrm{E}\left[W_{j}\left(U_{j t} ; \alpha_{0}\right)\right]+\mathrm{E}\left[Z_{j}\left(U_{j t}\right)\right], j=1,2 .
$$

It follows immediately that $\sqrt{n} \hat{\mathfrak{g}}(\hat{\alpha}) \stackrel{d}{\rightarrow} \mathcal{N}(0, \Omega)$ as $n \rightarrow \infty$..

Proof of Theorem 3. Consider a generic function $H\left(v_{1}, v_{2} ; \alpha\right):(0,1)^{2} \rightarrow \mathbb{R}$. We have by the triangle 
inequality,

$$
\begin{aligned}
& \left|\frac{1}{n} \sum_{t=1}^{n} H\left(\tilde{U}_{1 t}, \tilde{U}_{2 t} ; \hat{\alpha}\right)-\mathrm{E}\left[H\left(U_{1 t}, U_{2 t} ; \alpha_{0}\right)\right]\right| \\
\leq & \left|\frac{1}{n} \sum_{t=1}^{n} H\left(\tilde{U}_{1 t}, \tilde{U}_{2 t} ; \hat{\alpha}\right)-\frac{1}{n} \sum_{t=1}^{n} H\left(\tilde{U}_{1 t}, \tilde{U}_{2 t} ; \alpha_{0}\right)\right| \\
+ & \left|\frac{1}{n} \sum_{t=1}^{n} H\left(\tilde{U}_{1 t}, \tilde{U}_{2 t} ; \alpha_{0}\right)-\mathrm{E}\left[H\left(U_{1 t}, U_{2 t} ; \alpha_{0}\right)\right]\right| .
\end{aligned}
$$

First take $H\left(v_{1}, v_{2} ; \alpha\right)=l_{\alpha}\left(v_{1}, v_{2} ; \alpha\right) l_{\alpha}\left(v_{1}, v_{2} ; \alpha\right)^{T}$. It follows that under Condition 10, (A.8) converges to zero by the Dominated convergence Theorem. The second term, (A.9) vanishes asymptotically as a consequence of Proposition A.1(i) of Genest et al. (1995) or Lemma 1 of Chen and Fan (2005). It follows that $\hat{B}$ estimates $B$ consistently. Similarly, $\hat{G}$ estimate $G$ consistently under Condition 10. Lastly take $H\left(v_{1}, v_{2} ; \alpha\right)=\varphi_{t} \varphi_{t}^{T}$ with $\varphi_{t}$ given by (17). Using essentially the same argument, one can show that $\hat{\Omega}$ estimates $\Omega$ consistently under Condition 9 . The result of this theorem then follows readily from the asymptotic normality of $\hat{\mathfrak{g}}(\hat{\alpha})$ given in Theorem 2 .

Proof of Theorems 4 and 5. Under the regularity conditions given in the Appendix of Chen and Fan (2006), these theorems are direct results of Propositions 3.1 and 3.2 in the same paper. For brevity, the proofs are not reproduced here. 